\title{
Particle in a self-dual dyon background: hidden free nature, and exotic superconformal symmetry
}

\author{
Mikhail S. Plyushchay ${ }^{a}$ and Andreas Wipf ${ }^{b}$ \\ ${ }^{a}$ Departamento de Física, Universidad de Santiago de Chile, Casilla 307, Santiago 2, Chile \\ ${ }^{b}$ Theoretisch-Physikalisches Institut, Friedrich-Schiller-Universität Jena, Max-Wien-Platz 1, \\ D-07743 Jena, Germany \\ E-mails: mikhail.plyushchay@usach.cl,wipf@tpi.uni-jena.de
}

\begin{abstract}
We show that a non-relativistic particle in a combined field of a magnetic monopole and $1 / r^{2}$ potential reveals a hidden, partially free dynamics when the strength of the central potential and the charge-monopole coupling constant are mutually fitted to each other. In this case the system admits both a conserved Laplace-Runge-Lenz vector and a dynamical conformal symmetry. The supersymmetrically extended system corresponds then to a background of a self-dual or anti-self-dual dyon. It is described by a quadratically extended Lie superalgebra $D(2,1 ; \alpha)$ with $\alpha=1 / 2$, in which the bosonic set of generators is enlarged by a generalized Laplace-Runge-Lenz vector and its dynamical integral counterpart related to Galilei symmetry, as well as by the chiral $\mathbb{Z}_{2}$-grading operator. The odd part of the nonlinear superalgebra comprises a complete set of $24=2 \times 3 \times 4$ fermionic generators. Here a usual duplication comes from the $\mathbb{Z}_{2}$-grading structure, the second factor can be associated with a triad of scalar integrals - the Hamiltonian, the generator of special conformal transformations and the squared total angular momentum vector, while the quadruplication is generated by a chiral spin vector integral which exits due to the (anti)-self-dual nature of the electromagnetic background.
\end{abstract}

\section{Introduction}

Peculiar features of a classical or quantum system are usually associated with and reflected in its special symmetry properties. A well-known example is the conserved Laplace-Runge-Lenz vector, which explains the periodicity of the classical bound trajectories in the Kepler problem and the 'accidental' degeneracy of the bound states energy levels of the hydrogen atom [1]-6]. A different kind of example is provided by nonlinear integrable systems, in which soliton solutions exhibit particle-like properties in classical scattering processes. The robustness of solitons in these field systems is a consequence of the infinite number of conservation laws. In the inverse scattering method, solitons correspond to reflectionless potentials in the associated quantum problems [7. The reflectionless nature of soliton potentials can be linked, in turn, with a presence of a nontrivial Lax-Novikov quantum integral of motion which is a higher order differential operator. These peculiarities of the quantum mechanical soliton systems show up in a supersymmetric generalization, where they reveal a richer supersymmetry structure in comparison with that for the non-solitonic ones [8]. 
A charged particle in the field of a magnetic monopole exhibits a hidden free conical dynamics [9, 10]. In this aspect it resembles one-dimensional quantum mechanical reflectionless systems with their close relation to a free particle. A charge-dyon system, on the other hand, is characterized by the presence of the conserved Laplace-Runge-Lenz vector [11, similarly to the Kepler problem. The study of both the charge-monopole and charge-dyon systems, as well as their superextensions, has attracted a lot of attention in literature [9]-33].

This paper is devoted to the investigation of the rather exotic nonlinear superconformal structure of a particle in the field of the Dirac magnetic monopole accompanied by the field of the central $1 / r^{2}$-potential. Particular aspects of this system, including the supersymmetric one, have been investigated in earlier works [17, 18, 25]. In the present work we shall, however, emphasize the aspects related to the hidden symmetries. Namely, we first investigate in detail the spinless particle and show, that for a particular value of the strength of the central potential relative to the charge-monopole coupling, the system reveals a hidden partially free dynamics. As a result, besides the rotational and conformal symmetries, it will admit the conserved Laplace-Runge-Lenz vector as well as the associated dynamical (explicitly depending on time) vector integral related to the Galilei symmetry. Then we shall arrive at a related system from a different direction, by constructing the supersymmetric extension of the particle in an electromagnetic background field by incorporating spin degrees of freedom. We shall observe that for a (anti)-self-dual background the system admits a chiral spin integral of motion. As a result, we obtain a supersymmetric generalization of the original spinless system, which can be treated as a supersymmetric spinning particle in the field of a (anti)-self-dual dyon. The supersymmetric structure we obtain is rather unusual and unexpectedly rich. It incorporates the Laplace-Runge-Lenz and the associated dynamical vector integrals, the generators of conformal and rotational symmetries, and the chiral spin vector integral. They enter the resulting partially nonlinear (quadratic) superalgebra with 24 quantum fermionic generators, which represents a certain extension of the superconformal $D(2,1 ; \alpha)$ symmetry 25 , with a particular value of the parameter $\alpha=1 / 2$.

In the following section we investigate the spinless particle and in particular the special case characterized by the presence of the conserved Laplace-Runge-Lenz vector and a partially free dynamics. In the third section we construct the supersymmetric extension of the system, and study its nonlinear superconformal structure both at the classical and quantum levels. The last, fourth section includes a summary and concluding remarks.

\section{Spinless case}

Consider a non-relativistic particle of charge $e$ and mass $m$ in a combined field of a magnetic monopole1 $1, \vec{B}=g \vec{r} / r^{3}$, and central potential $U(r)$. It is described by the Hamiltonian

$$
H=\frac{1}{2 m} \vec{\Pi}^{2}+U(r)
$$

and Poisson brackets

$$
\left\{r_{i}, r_{j}\right\}=0, \quad\left\{r_{i}, \Pi_{j}\right\}=\delta_{i j}, \quad\left\{\Pi_{i}, \Pi_{j}\right\}=e \epsilon_{i j k} B_{k} .
$$

The equations of motion for the position vector and kinetic-momentum read

$$
\dot{\vec{r}}=\frac{1}{m} \vec{\Pi}, \quad \dot{\vec{\Pi}}=-\frac{\nu}{m r^{3}} \vec{L}-U^{\prime}(r) \vec{n},
$$

\footnotetext{
${ }^{1}$ We use the units $c=\hbar=1$.
} 
where $\nu=e g, \vec{n}=\vec{r} / r, \vec{L}=\vec{r} \times \vec{\Pi}$. From here one finds

$$
\dot{\vec{n}}=\frac{1}{m r^{2}} \vec{L} \times \vec{n}, \quad \dot{\vec{L}}=\frac{\nu}{m r^{2}} \vec{L} \times \vec{n},
$$

and

$$
\frac{d}{d t} \vec{r}^{2}=\frac{2}{m} \vec{\Pi} \cdot \vec{r}, \quad \frac{d}{d t}(\vec{\Pi} \cdot \vec{r})=2 H-\left(2 U+r U^{\prime}\right) .
$$

From (2.4) it follows that the Poincaré vector

$$
\vec{J}=\vec{L}-\nu \vec{n}, \quad \text { with } \quad \vec{J}^{2}=\vec{L}^{2}+\nu^{2} \geq \nu^{2},
$$

is an integral of motion for any choice of the central potential. It is just the angular momentum of the system:

$$
\left\{J_{i}, J_{j}\right\}=\epsilon_{i j k} J_{k}, \quad\left\{J_{i}, n_{j}\right\}=\epsilon_{i j k} n_{k}, \quad\left\{J_{i}, r\right\}=\left\{J_{i}, \Pi_{r}\right\}=0,
$$

where $\Pi_{r}=\vec{\Pi} \cdot \vec{n}$ is the radial component of the kinetic momentum and we also have $\left\{r, \Pi_{r}\right\}=1$. In terms of the variables $\vec{J}, \vec{n}, r$ and $\Pi_{r}$ the Hamiltonian takes the form

$$
H=\frac{1}{2 m}\left(\Pi_{r}^{2}+\frac{(\vec{J} \times \vec{n})^{2}}{r^{2}}\right)+U(r), \quad \text { with } \quad(\vec{J} \times \vec{n})^{2}=\vec{J}^{2}-\nu^{2}
$$

The vectors $\vec{n}$ and $\vec{L}$ precess around the conserved angular momentum $\vec{J}$ with the same frequency,

$$
\dot{\vec{n}}=\frac{1}{m r^{2}} \vec{J} \times \vec{n}, \quad \dot{\vec{L}}=\frac{1}{m r^{2}} \vec{J} \times \vec{L} .
$$

Hence the trajectory of the particle lies on the cone defined by $\vec{J} \cdot \vec{n}=-\nu$ with vertex in $r=0$ and symmetry axis oriented along the vector $\vec{J}$. For $U(r)=0$ the particle moves on geodesics on the cone [9, 10], and like a free particle $(\nu=0)$ is characterized by a conformal symmetry [34-39]. This symmetry survives under switching on the inverse square potential

$$
U(r)=\frac{\lambda}{r^{2}}
$$

In this case the scalar $\vec{\Pi} \cdot \vec{r}$ is subject to a simple dynamics, $\frac{d}{d t}(\vec{\Pi} \cdot \vec{r})=2 H$. As a consequence, the dilatation generator

$$
D=\vec{\Pi} \cdot \vec{r}-2 t H
$$

is an explicitly time-dependent dynamical integral of motion: $\frac{d}{d t} D=\frac{\partial}{\partial t} D+\{D, H\}=0$. The first equation from (2.5) implies then that another dynamical integral of motion exists:

$$
K=2 m r^{2}-4 t D-4 t^{2} H .
$$

It is the generator of special conformal transformations.

From now on, we shall consider the potential (2.10) characterized by the presence of the two dynamical integrals of motion $D$ and $K$, and assume that $\lambda>0$ to avoid the problem of the fall to the center $r=0$. We shall see that the system admits an even richer symmetry structure when the relation $\lambda=\nu^{2} / 2 m$ between the couplings holds true. This particular choice of couplings is also distinguished by the supersymmetric extension of the system. 
The minimal distance of the particle from the force center corresponds to the instant $t_{0}$ for which $(\vec{\Pi} \cdot \vec{r})\left(t_{0}\right)=0$, see Eq. (2.5). Taking into account relation $\vec{L}^{2}=2 m r^{2} H-(\vec{\Pi} \cdot \vec{r})^{2}-\mu^{2}$, where $\mu^{2}=2 m \lambda$, one finds that $r_{\text {min }}^{2}=\left(\vec{J}^{2}-\nu^{2}+\mu^{2}\right) /(2 m H)$. Now we decompose the unit vector $\vec{n}$ into the parts $\vec{n}_{\|}$and $\vec{n}_{\perp}$ parallel and perpendicular to the total angular momentum. Due to (2.6) the former is time independent, $\vec{n}_{\|}=-\nu \vec{J} / \vec{J}^{2}$, and this implies $\vec{n}_{\perp}^{2}=\left(\vec{J}^{2}-\nu^{2}\right) / \vec{J}^{2}$. Thus the latter can be written as

$$
\vec{n}_{\perp}(t)=\vec{n}_{\perp}\left(t_{0}\right) \cos \varphi(t)+\frac{1}{J} \vec{J} \times \vec{n}_{\perp}\left(t_{0}\right) \sin \varphi(t) .
$$

Since $\dot{\vec{n}}_{\perp}=\dot{\vec{n}}$ the first relation in (2.9) implies $\dot{\varphi}=J /\left(m r^{2}\right)$. Employing the relations $1 / r^{2}=$ $2 m H /\left[(\vec{\Pi} \cdot \vec{r})^{2}+\vec{L}^{2}+\mu^{2}\right]$ and $2 H=\frac{d}{d t}(\vec{\Pi} \cdot \vec{r})$, we obtain $d \varphi=J d(\vec{\Pi} \cdot \vec{r}) /\left[(\vec{\Pi} \cdot \vec{r})^{2}+\vec{L}^{2}+\mu^{2}\right]$. This yields the evolution law for the angle $\varphi$ in the plane orthogonal to the angular momentum,

$$
\varphi(t)=\frac{J}{\sqrt{\vec{L}^{2}+\mu^{2}}} \arctan \left(\vec{\Pi} \cdot \vec{r} / \sqrt{\vec{L}^{2}+\mu^{2}}\right)+\text { const. }
$$

For $t_{0}=0$ and a vanishing integration constant the angle vanishes when $r$ is minimal, $\vec{\Pi} \cdot \vec{r}=2 H t$, and $r^{2}(t)=r_{\min }^{2}+2 H t^{2} / m$, such that

$$
\begin{gathered}
\varphi(t)=\frac{J}{\sqrt{J^{2}-\nu^{2}+\mu^{2}}} \arctan \left(\frac{2 H t}{\sqrt{J^{2}-\nu^{2}+\mu^{2}}}\right), \\
\vec{n}(t)=-\nu \frac{1}{J^{2}} \vec{J}+\vec{n}_{\perp}(t), \quad \vec{n}_{\perp}(t)=\left(\vec{n}(0)+\nu \frac{1}{J^{2}} \vec{J}\right) \cos \varphi(t)+\frac{1}{J} \vec{J} \times \vec{n}(0) \sin \varphi(t) .
\end{gathered}
$$

The scattering angle of the trajectory projected onto the plane orthogonal to $\vec{J}$ is

$$
\Delta \varphi-\pi=\int_{-\infty}^{+\infty} \dot{\varphi} d t-\pi=\pi\left(\frac{J}{\sqrt{J^{2}-\nu^{2}+\mu^{2}}}-1\right)
$$

In general it depends on the value of the angular momentum $J$. Only in the exceptional case when $\mu^{2}=\nu^{2}$ we have $\Delta \varphi-\pi=0$ for all $J^{2} \geq \nu^{2}$.

From now on we restrict our analysis to the special case $\mu^{2}=\nu^{2}$, for which the Hamiltonian can be presented in the two equivalent forms

$$
H=\frac{1}{2 m}\left(\vec{\Pi}^{2}+\frac{\nu^{2}}{r^{2}}\right)=\frac{1}{2 m}\left(\Pi_{r}^{2}+\frac{\vec{J}^{2}}{r^{2}}\right) .
$$

Only for this particular value of the parameter $\lambda$ the central potential compensates exactly the term $-\nu^{2} / r^{2}$ appearing in the centrifugal term $(\vec{J} \times \vec{n})^{2} / r^{2}$ of the charge-monopole Hamiltonian, see Eq. (2.8).

The cos- and sin-functions, which enter the precession law of the unit vector, simplify (only) in this case to elementary functions, $\cos \varphi(t)=1 / \sqrt{1+\tau^{2}}, \sin \varphi(t)=\tau / \sqrt{1+\tau^{2}}$, where we introduced the dimensionless time variable $\tau=2 \mathrm{Ht} / \mathrm{J}$. The distance of the particle from the force center varies as $r(t)=J \sqrt{1+\tau^{2}} / \sqrt{2 m H}$, and we get

$$
r_{\min }=r(0)=\frac{J}{\sqrt{2 m H}} .
$$


The evolution law for the particle's coordinate vector can then be written as

$$
\vec{r}(t)=\vec{r}(0)+\frac{J}{\sqrt{2 m H}}\left(\frac{\nu}{J^{2}} \vec{J}\left(1-\sqrt{1+\tau^{2}}\right)+\frac{1}{J}(\vec{J} \times \vec{n}(0)) \tau\right) .
$$

The projected motion of the particle in the plane orthogonal to the angular momentum is that of a free particle: it moves along a straight line with constant velocity.

Peculiar properties of a dynamical system both at the classical and quantum levels are in many cases associated with the presence of hidden symmetries. This happens also in the special case of the system (2.18). Indeed, consider the Laplace-Runge-Lenz-vector

$$
\vec{G}=\vec{\Pi} \times \vec{J}+\kappa \vec{n},
$$

where $\kappa$ is a constant with the dimension of a mass. In the general case with central potential (2.8) its dynamics is given by

$$
\frac{d}{d t} \vec{G}=\vec{L} \times \vec{n}\left(U^{\prime}+\frac{\nu^{2}}{m r^{3}}+\frac{\kappa}{m r^{2}}\right) .
$$

The vector is an integral of motion only for central potentials of the form

$$
U(r)=\frac{\nu^{2}}{2 m r^{2}}+\frac{\kappa}{m r},
$$

that is for a linear combination of the Kepler potential and the particular potential (2.10) with

$$
2 m \lambda=\mu^{2}=\nu^{2}
$$

The case $\kappa=0$ is characterized by the presence of the additional conformal symmetry associated with dynamical integrals $D$ and $K$. The particular system (2.18) therefore admits the additional integral of motion

$$
\vec{G}=\vec{\Pi} \times \vec{J} .
$$

This, particularly, can easily be seen from the equation of motion

$$
\dot{\vec{\Pi}}=-\frac{\nu}{m r^{3}} \vec{J}
$$

which holds for relation (2.24) between the couplings, see Eqs. (2.3) and (2.6).

From relations (2.25) and (2.6) one also finds

$$
\vec{G} \cdot \vec{r}=J^{2}-\nu^{2} .
$$

Eq. (2.20) in particular means, that the vector $\vec{J} \times \vec{n}(0)$ is oriented along the integral

$$
\vec{G} \times \vec{J}=-J^{2} \vec{\Pi}-\nu \Pi_{r} \vec{J} .
$$

The conserved vectors $\vec{J}, \vec{G}$ and $\vec{G} \times \vec{J}$ form an orthogonal basis, and in addition to (2.27), the projections of $\vec{r}$ and $\vec{\Pi}$ onto these vectors are

$$
\begin{gathered}
\vec{r} \cdot \vec{J}=-\nu r, \quad \vec{r} \cdot(\vec{G} \times \vec{J})=-r \Pi_{r}\left(J^{2}-\nu^{2}\right), \\
\vec{\Pi} \cdot \vec{G}=0, \quad \vec{\Pi} \cdot(\vec{G} \times \vec{J})=-\vec{G}^{2}, \quad \vec{\Pi} \cdot \vec{J}=-\nu \Pi_{r} .
\end{gathered}
$$


In addition, note that

$$
\vec{G}^{2}=2 m H\left(J^{2}-\nu^{2}\right), \quad(\vec{G} \times \vec{J})^{2}=\vec{G}^{2} J^{2} .
$$

The angle between the vectors $\vec{n}(t)$ and $\vec{J}$ is given by $\cos \theta=-\nu J^{-1}$. Taking into account Eq. (2.19), one finds that relation (2.27) can be written in the equivalent form

$$
\vec{G} \cdot(\vec{r}(t)-\vec{r}(0))=0 .
$$

The trajectory of the particle lies, therefore, in the plane orthogonal to $\vec{G}$. We conclude that the trajectory is given by intersection of the cone $\vec{J} \cdot \vec{n}=-\nu$ with the specified plane. It has a form of a hyperbola, whose projection onto the plane orthogonal to $\vec{J}$ is a straight line parallel to the conserved vector $\vec{G} \times \vec{J}$. The projected coordinate of the particle evolves with constant speed along this line. The equation of hyperbola can be presented in the form

$$
\frac{(\vec{r} \cdot \vec{J})^{2}}{\nu^{2} J^{2}}-\frac{(\vec{r} \cdot(\vec{G} \times \vec{J}))^{2}}{G^{2} J^{2}\left(J^{2}-\nu^{2}\right)}=\frac{1}{2 m H} .
$$

Since the conserved vectors $\vec{J}, \vec{G}$ and $\vec{G} \times \vec{J}$ form the complete orthogonal set in the 3-dimensional space, one finds

$$
\vec{r}=\frac{1}{2 m H} \vec{G}-\frac{\nu r}{J^{2}} \vec{J}-\frac{\vec{r} \cdot \vec{\Pi}}{2 m H J^{2}} \vec{G} \times \vec{J}
$$

where the relations

$$
(\vec{r} \cdot \vec{\Pi})(t)=(\vec{r} \cdot \vec{\Pi})(0)+2 H t, \quad r^{2}(t)=r^{2}(0)+\frac{2}{m}(\vec{r} \cdot \vec{\Pi})(0) t+\frac{2}{m} H t^{2}
$$

finally determine the evolution of $\vec{r}(t)$. One obtains the same law as in (2.20).

In addition to the scalar integrals $D$ and $K$, explicitly depending on time, the first equation in (2.3) and Eq. (2.25) allow us to construct an analogous, dynamical vector integral depending explicitly on time,

$$
\vec{R}=\vec{r} \times \vec{J}-\frac{t}{m} \vec{G}
$$

It satisfies the relations

$$
2 m H \vec{R}=D \vec{G}+\vec{G} \times \vec{J},
$$

and we also get

$$
\begin{gathered}
\vec{R} \cdot \vec{J}=0, \quad \vec{R} \cdot(\vec{G} \times \vec{J})=J^{2}\left(J^{2}-\nu^{2}\right), \\
\vec{R} \cdot \vec{G}=\left(J^{2}-\nu^{2}\right) D, \quad \vec{R}^{2}=\frac{1}{2 m}\left(J^{2}-\nu^{2}\right) K .
\end{gathered}
$$

In the liming case $\nu \rightarrow 0$ corresponding to a free particle, one gets

$$
\vec{R} \rightarrow D\left(\vec{r}-\frac{t}{m} \vec{p}\right)-\frac{K}{2 m} \vec{p}
$$

In more detail, at $g=\nu=0$, the mechanical (or kinetic) momentum $\vec{\Pi}$ turns into the canonical momentum $\vec{p}$ with Poisson-commuting components, $\left\{p_{i}, p_{j}\right\}=0$. At the same time the angular momentum $\vec{J}$ becomes the orbital angular momentum and the system transforms into a free particle with $H=\vec{p}^{2} / 2 m$. In accordance with Eq. (2.28), the integral $\vec{G} \times \vec{J}$ reduces to canonical momentum vector $\vec{p}$ multiplied by the integral $-\vec{L}^{2}$. The Laplace-Runge-Lenz vector $\vec{G}$ itself 
reduces to $\vec{p} \times \vec{L}=\vec{p}^{2} \vec{r}-(\vec{p} \cdot \vec{r}) \vec{p}$. Note that free particle system possesses the additional dynamical integral $\vec{N}=\vec{r}-\vec{p} t / m$ that coincides with $\vec{r}(0)$, and is a generator of Galilei boosts. It is interesting to compare the free particle relations $\vec{N} \cdot \vec{p}=D$ and $2 m \vec{N}^{2}=K$ with (2.39). The integral $\vec{p} \times \vec{L}$ can be written in terms of the dynamical integrals $\vec{N}$ and $D$ as $\vec{p} \times \vec{L}=2 m H \vec{N}-D \vec{p}$, which can be compared with the limit relation (2.37) for the dynamical vector integral $\vec{R}$. The trajectory of the free particle is a straight line along the vector $\vec{p}$ that passes though the point $\vec{r}(0)=\vec{N}$ and lies in the plane orthogonal to $\vec{L}$. Switching on the magnetic monopole field and at the same time the scalar potential $U(r)=\nu^{2} / 2 m r^{2}$ results in 'lifting' and deforming the straight line into the hyperbola given by the intersection of the magnetic monopole cone $\vec{J} \cdot \vec{n}=-\nu$ with a plane orthogonal to $\vec{G}$ and passing through the point $\vec{r}(0)$.

From (2.31) it follows that the Hamiltonian of the system can be presented in terms of the angular momentum and Laplace-Runge-Lenz vector,

$$
H=\frac{1}{2 m} \frac{G^{2}}{J^{2}-\nu^{2}}
$$

The latter satisfies the Poisson bracket relation

$$
\left\{G_{i}, G_{j}\right\}=-2 m H \epsilon_{i j k} J_{k} .
$$

As $H>0$, one defines the vector

$$
\vec{V}=\frac{\vec{G}}{\sqrt{2 m H}} .
$$

This re-scaled Laplace-Runge-Lenz vector together with $\vec{J}$ generate the $s o(3,1)$ Lorentz algebra,

$$
\left\{J_{i}, J_{j}\right\}=\epsilon_{i j k} J_{k}, \quad\left\{V_{i}, V_{j}\right\}=-\epsilon_{i j k} J_{k}, \quad\left\{J_{i}, V_{j}\right\}=\epsilon_{i j k} V_{k} .
$$

The quantities $\mathcal{C}_{1}=\vec{V}^{2}-\vec{J}^{2}$ and $\mathcal{C}_{2}=\vec{J} \cdot \vec{V}$ are two independent Casimirs of the $s o(3,1)$ algebra (2.44), $\left\{\mathcal{C}_{a}, J_{i}\right\}=\left\{\mathcal{C}_{a}, V_{i}\right\}=0, a=1,2$, which have here the values $\mathcal{C}_{1}=\nu^{2}$ and $\mathcal{C}_{2}=0$. In terms of the complex combinations $\mathcal{L}_{j}^{ \pm}=\frac{1}{2}\left(J_{j} \pm i V_{j}\right)$, we have

$$
\left\{\mathcal{L}_{i}^{+}, \mathcal{L}_{j}^{+}\right\}=\epsilon_{i j k} \mathcal{L}_{k}^{+}, \quad\left\{\mathcal{L}_{i}^{-}, \mathcal{L}_{j}^{-}\right\}=\epsilon_{i j k} \mathcal{L}_{k}^{-}, \quad\left\{\mathcal{L}_{i}^{+}, \mathcal{L}_{j}^{-}\right\}=0 .
$$

In conclusion of this section, let us note that we have identified additional integrals of motion for particular central potential by first analyzing the scattering of the particle. The acceleration points in the direction of $\vec{J}$ only if $U(r)=\nu^{2} /\left(2 m r^{2}\right)+$ const, i.e. exactly for the particular central potential we have studied. For this potential $\vec{G}=\vec{\Pi} \times \vec{J}$ is an integral of motion. The acceleration of $\vec{r}$, projected on the integral $\vec{J} \times \vec{G}$, is zero and this reveals a hidden partially free dynamics of the particle. The relation $\frac{d^{3}}{d t^{3}}\left(r^{2}\right)=0$ is equivalent to the condition that $K$ is a dynamical integral of motion. Since $\vec{r} \cdot \vec{J}=-\nu r$, this reduces to the equation $\frac{d^{3}}{d t^{3}}(\vec{r} \cdot \vec{J})^{2}=0$. The last relation means that the acceleration of the particle along $\vec{J}$ is constant, and from here we recover the hyperbolic form of the trajectory.

Note that the system (2.18) corresponds to a spinless part of the model [17] at the "points of higher symmetry" $\lambda^{2}=\nu^{2}$. It was discussed in [25], where a special hyperbolic trajectory was also identified and associated with the presence of the Laplace-Runge-Lenz vector. However, there the dynamical integral (2.36) was not considered. As we shall see below, both the vector integrals $\vec{G}$ and $\vec{R}$ (more precisely, their analogs incorporating spin degrees of freedom) will play the key role in a nonlinear supeconformal structure of the superextended version of the system.

In the next section we find a supersymmetric extension of the system (2.18) by exploiting its particular symmetry properties. 


\section{Supersymmetric extension: particle in (anti)-self-dual dyon background}

To construct a supersymmetric generalization of the system, we introduce four Grassmann variables $\xi_{a}$, where $a=0, i$, and $i=1,2,3$, with Poisson brackets

$$
\left\{\xi_{a}, \xi_{b}\right\}=-i \delta_{a b}
$$

Their quantum analogs are given by Euclidean gamma-matrices $\gamma_{a}, \hat{\xi}_{0}=\frac{1}{\sqrt{2}} \gamma_{0}, \hat{\xi}_{i}=\frac{1}{\sqrt{2}} \gamma_{i}$, realized, for example, via two sets of the Pauli matrices,

$$
\gamma_{0}=\tau_{1} \otimes 1=\left(\begin{array}{ll}
\mathbf{0} & \mathbf{1} \\
\mathbf{1} & \mathbf{0}
\end{array}\right), \quad \gamma_{i}=\tau_{2} \otimes \sigma_{i}=\left(\begin{array}{cc}
\mathbf{0} & -i \sigma_{i} \\
i \sigma_{i} & \mathbf{0}
\end{array}\right)
$$

We distinguish the values $a=0$ and $a=1,2,3$ since in the model we shall obtain the $\xi_{0}$ and $\xi_{i}$ will have different transformation properties under the spatial rotations. The operators $\hat{\xi}_{a}$ anti-commute with

$$
\Gamma \equiv \gamma_{5}=\tau_{3} \otimes 1=\left(\begin{array}{cc}
\mathbf{1} & \mathbf{0} \\
\mathbf{0} & \mathbf{- 1}
\end{array}\right), \quad \Gamma^{2}=1,
$$

which at the quantum level is identified as a $Z_{2}$-grading operator of the superalgebraic structure. We introduce also the chiral projectors

$$
\mathcal{T}_{ \pm}=\frac{1}{2}\left(1 \pm \gamma_{5}\right)=\frac{1}{2}\left(1 \pm \tau_{3}\right) \otimes 1, \quad \mathcal{T}_{+}+\mathcal{T}_{-}=1, \quad \mathcal{T}_{+} \mathcal{T}_{-}=0
$$

In terms of the Grassmann variables $\xi_{i}$ and $\xi_{0}$, one defines the chiral spin vectors:

$$
\mathcal{S}_{i}^{ \pm}=\frac{1}{2}\left(\mathcal{S}_{i} \pm \mathcal{V}_{i}\right), \quad \text { where } \quad \mathcal{S}_{i}=-\frac{i}{2} \epsilon_{i j k} \xi_{j} \xi_{k}, \quad \mathcal{V}_{i}=-i \xi_{0} \xi_{i}
$$

They generate the $s o(4)=s o(3) \oplus s o(3)$ algebra,

$$
\left\{\mathcal{S}_{i}^{+}, \mathcal{S}_{j}^{+}\right\}=\epsilon_{i j k} \mathcal{S}_{k}^{+}, \quad\left\{\mathcal{S}_{i}^{-}, \mathcal{S}_{j}^{-}\right\}=\epsilon_{i j k} \mathcal{S}_{k}^{-}, \quad\left\{\mathcal{S}_{i}^{+}, \mathcal{S}_{j}^{-}\right\}=0 .
$$

The $\mathcal{S}_{i}^{+}$has the following Poisson brackets with the $\xi_{a}$ :

$$
\left\{\mathcal{S}_{i}^{+}, \xi_{j}\right\}=\frac{1}{2}\left(\epsilon_{i j k} \xi_{k}-\xi_{0} \delta_{i j}\right), \quad\left\{\mathcal{S}_{i}^{+}, \xi_{0}\right\}=\frac{1}{2} \xi_{i}
$$

Analogous relations for $\mathcal{S}_{i}^{-}$are obtained from (3.7) by the change $\xi_{0} \rightarrow-\xi_{0}$.

The quantum analogs of $\mathcal{S}_{i}^{ \pm}$contain the chiral projectors, $\hat{\mathcal{S}}_{i}^{ \pm}=\mathcal{T}_{ \pm} \hat{S}_{i}$, where $\hat{S}_{i}=\hat{\mathcal{S}}_{i}^{+}+\hat{\mathcal{S}}_{i}^{-}=$ $1 \otimes \frac{1}{2} \sigma_{i}$, and, particularly, the quantum analog of the third relation from $(\underline{3.6}),\left[\hat{\mathcal{S}}_{i}^{+}, \hat{\mathcal{S}}_{j}^{-}\right]=0$, is just a trivial consequence of the opposite chiralities of $\hat{\mathcal{S}}_{i}^{+}$and $\hat{\mathcal{S}}_{i}^{-}$.

Now we consider a particle with charge $e$ propagating in an electric and magnetic fields described by a vector potential $A_{i}(\vec{r})$ and a scalar potential $A_{0}(\vec{r})$, and consider a Grassmannodd classical quantity

$$
\Theta_{0}=\Pi_{i} \xi_{i}+\phi(\vec{r}) \xi_{0},
$$

where $\phi(\vec{r})=e A_{0}(\vec{r})$ and $\Pi_{i}(\vec{r})=p_{i}-e A_{i}(\vec{r})$ are the components of the kinetic momentum $\vec{\Pi}$. From here on we set $2 m=1$. We have $\left\{\Pi_{i}, \Pi_{j}\right\}=\epsilon_{i j k} \mathcal{B}_{k}$ and $\left\{\Pi_{i}, \phi\right\}=\mathcal{E}_{i}$ with $\mathcal{B}_{i}=e B_{i}$ and $\mathcal{E}_{i}=e E_{i}$. Here $B_{i}=\epsilon_{i j k} \partial_{j} A_{k}$ and $E_{i}=-\partial_{i} A_{0}$ are the background magnetic and electric fields, whose forms are not further specified at the moment. 
The Grassmann-even quantity $\mathcal{H}$ generated by $\Theta_{0}$,

$$
\begin{gathered}
\left\{\Theta_{0}, \Theta_{0}\right\}=-i \mathcal{H}, \\
\mathcal{H}=\Pi_{i}^{2}+\phi^{2}-2\left(\mathcal{S}_{i}^{+}\left(\mathcal{B}_{i}-\mathcal{E}_{i}\right)+\mathcal{S}_{i}^{-}\left(\mathcal{B}_{i}+\mathcal{E}_{i}\right)\right),
\end{gathered}
$$

is readily identified as a Pauli type, second order in $p_{i}$ Hamiltonian. With such an interpretation, $\Theta_{0}$ can be considered as a classical analog of the stationary $(\partial / \partial t \rightarrow 0)$, first order in $p_{i}$ Dirac operator. From the generalized, graded Jacobi identity $3\left\{\Theta_{0},\left\{\Theta_{0}, \Theta_{0}\right\}\right\}=0$ it follows at once that $\left\{\Theta_{0}, \mathcal{H}\right\}=0$, and so, $\Theta_{0}$ can be treated as a supercharge for the system with the Hamiltonian $\mathcal{H}$. Eq. (3.10) shows that independently from the rotational properties of potentials $A_{i}$ and $A_{0}$, there are two special case 22: self-dual, when $\mathcal{B}_{i}=\mathcal{E}_{i}$, and anti-self-dual, $\mathcal{B}_{i}=-\mathcal{E}_{i}$. As follows from the last relation in (3.6), in these two cases we have additional Grassmann-even integrals of motion, $\mathcal{S}_{i}^{+}$, or $\mathcal{S}_{i}^{-}$, respectively. Since with any anti-self-dual background one can associate the corresponding self-dual background just by changing $A_{0} \rightarrow-A_{0}, A_{i} \rightarrow A_{i}$, one can restrict the consideration to the self-dual case. Then the Hamiltonian reduces to

$$
\mathcal{H}=\Pi_{i}^{2}+\phi^{2}-4 \mathcal{S}_{i}^{-} \mathcal{B}_{i}
$$

and in addition to the supercharge $\Theta_{0}$ we have the integrals of motion $\mathcal{S}_{i}^{+}$generating an so(3) symmetry. As the Poisson brackets of integrals of motion are also integrals of motion, we get three more integrals

$$
\Theta_{i} \equiv \epsilon_{i j k} \Pi_{j} \xi_{k}+\phi \xi_{i}-\Pi_{i} \xi_{0}
$$

where $\Theta_{i}=2\left\{\mathcal{S}_{i}^{+}, \Theta_{0}\right\}$. Let us stress that the integrals $\Theta_{i}$ and $\Theta_{0}$ form the set with the same transformation properties with respect to the $s o(3)$ generators $\mathcal{S}_{i}^{+}$as the set formed by the basic Grassmann variables $\xi_{i}$ and $\xi_{0}$,

$$
\left\{\mathcal{S}_{i}^{+}, \Theta_{0}\right\}=\frac{1}{2} \Theta_{i}, \quad\left\{\mathcal{S}_{i}^{+}, \Theta_{j}\right\}=\frac{1}{2}\left(\epsilon_{i j k} \Theta_{k}-\delta_{i j} \Theta_{0}\right) .
$$

Employing (3.13), together with the conservation of $\mathcal{S}_{i}^{+}$and the graded Jacobi identity $-\left\{\Theta_{0},\left\{\mathcal{S}_{i}^{+}, \Theta_{0}\right\}\right\}+\left\{\mathcal{S}_{i}^{+},\left\{\Theta_{0}, \Theta_{0}\right\}\right\}+\left\{\Theta_{0},\left\{\Theta_{0}, \mathcal{S}_{i}^{+}\right\}\right\}=0$, we find that $\Theta_{i}$ and $\Theta_{0}$ Poissoncommute,

$$
\left\{\Theta_{0}, \Theta_{i}\right\}=0
$$

similarly to $\xi_{i}$ and $\xi_{0}$. Once again using the Jacobi identity and relations (3.13) and (3.9), we get

$$
\left\{\Theta_{i}, \Theta_{j}\right\}=-i \delta_{i j} \mathcal{H}
$$

Thus, postulating the supercharge (3.8) and choosing the (anti)self-dual electromagnetic background, we have got the second order in momenta $p_{i}$ system possessing the $s o(3) \cong s u(2)$ symmetry, whose generators $\mathcal{S}_{i}^{+}$give rise to the extension of the $N=1$ supersymmetry (3.9) up to the $N=4$ supersymmetry (3.9), (3.14), (3.15). Note here that for the first time it was showed in [40] that the $N=4$ supersymmetry for a particle in three-dimensional space necessarily implies the self-duality of the electromagnetic background, see also [17, 25, 26].

Up to this point we did not assume any particular properties of the background field with respect to the spatial rotations. Suppose now that electric field is spherically symmetric and choose $\phi=\phi(r)$. Then $\overrightarrow{\mathcal{E}}=-\vec{r} \phi^{\prime}(r) / r=\overrightarrow{\mathcal{B}}$, and the Maxwell equation $\partial_{i} \mathcal{B}_{i}=0$ for $\vec{r} \neq 0$ fixes the magnetic field to be that of the magnetic monopole, $\overrightarrow{\mathcal{B}}=\nu \vec{r} / r^{3}$. We arrive therefore at

\footnotetext{
${ }^{2}$ We are not interested here in another special case corresponding to homogeneous electric and magnetic fields.
} 
the electromagnetic background of the self-dual dyon characterized in general case by the scalar potential $\phi(r)=\kappa+\nu / r$, where $\kappa$ is a constant. Hamiltonian (3.11) is then a supersymmetric generalization of the spinless case given by the potential (2.23). We are interested in the supersymmetric generalization of the special case characterized by a hidden partially free particle dynamics. So, we put $\kappa=0$, and the Hamiltonian of the system takes the form (3.11) with $\phi=\nu / r$,

$$
\mathcal{H}=\Pi_{i}^{2}+\frac{\nu^{2}}{r^{2}}-4 \nu \frac{1}{r^{3}} \mathcal{S}_{i}^{-} r_{i}
$$

Its Grassmann-free, spinless part coincides with the Hamiltonian (2.18), which possesses the dynamical conformal symmetry, and one can expect that the total symmetry of (3.16) has to be a supersymmetric extension of that of the spinless system (2.18). Having in mind this perspective, we shall show now how an exotic nonlinear (quadratic) superconformal algebra for (3.16) appears by exploiting our knowledge about the symmetries of the system (2.18).

First, we find that the total angular momentum $\mathcal{J}_{i}=J_{i}+\mathcal{S}_{i}$ is an integral of motion of the system (3.16), where $J_{i}=(\vec{r} \times \vec{\Pi})_{i}-\nu n_{i}$. With respect to it, the $\xi_{i}$ form a vector and $\xi_{0}$ a scalar, and the Grassmann-odd supercharges $\Theta_{i}$ and $\Theta_{0}$ have exactly the same rotational properties. The $\mathcal{S}_{i}^{+}$is the Grassmann-even vector integral. As a result we find that the total angular momentum $\mathcal{J}_{i}$ and the chiral spin $\mathcal{S}_{i}^{+}$generate the bosonic $s o(4)=s o(3) \oplus s o(3)$ symmetry,

$$
\left\{\mathcal{Y}_{i}^{+}, \mathcal{Y}_{j}^{+}\right\}=\epsilon_{i j k} \mathcal{Y}_{k}^{+}, \quad\left\{\mathcal{Y}_{i}^{-}, \mathcal{Y}_{j}^{-}\right\}=\epsilon_{i j k} \mathcal{Y}_{k}^{-}, \quad\left\{\mathcal{Y}_{i}^{+}, \mathcal{Y}_{j}^{-}\right\}=0
$$

where

$$
\mathcal{Y}_{i}^{+} \equiv \mathcal{S}_{i}^{+}, \quad \mathcal{Y}_{i}^{-} \equiv \mathcal{J}_{i}-\mathcal{S}_{i}^{+}
$$

The Poisson bracket relations for the supercharges $\Theta_{a}$ with the bosonic integrals $\mathcal{Y}_{i}^{ \pm}$are

$$
\left\{\mathcal{Y}_{i}^{ \pm}, \Theta_{0}\right\}= \pm \frac{1}{2} \Theta_{i}, \quad\left\{\mathcal{Y}_{i}^{ \pm}, \Theta_{j}\right\}=\frac{1}{2}\left(\epsilon_{i j k} \Theta_{k} \mp \delta_{i j} \Theta_{0}\right)
$$

As in the spinless case, the superextended system also possesses dynamical integrals corresponding to scale- and special conformal transformations. The corresponding Grassmann-even dynamical scalar integrals are

$$
\mathcal{D}=\vec{\Pi} \cdot \vec{r}-2 \mathcal{H} t, \quad \mathcal{K}=\vec{r}^{2}-4 \mathcal{D} t-4 \mathcal{H} t^{2} .
$$

Indeed one finds $\frac{d}{d t} \mathcal{I}=\{\mathcal{I}, \mathcal{H}\}+\frac{\partial}{\partial t} \mathcal{I}=0$, where $\mathcal{I}=\mathcal{D}, \mathcal{K}$. Together with the Hamiltonian, they generate the $s o(2,1)$ algebra,

$$
\{\mathcal{D}, \mathcal{H}\}=2 \mathcal{H}, \quad\{\mathcal{D}, \mathcal{K}\}=-2 \mathcal{K}, \quad\{\mathcal{K}, \mathcal{H}\}=4 \mathcal{D} .
$$

In terms of the standard basis

$$
\mathbb{J}_{0}=\frac{1}{4}(\mathcal{H}+\mathcal{K}), \quad \mathbb{J}_{1}=\frac{1}{4}(\mathcal{H}-\mathcal{K}), \quad \mathbb{J}_{2}=\frac{1}{2} \mathcal{D},
$$

the $s o(2,1)$-structure is manifest, $\left\{\mathbb{J}_{0}, \mathbb{J}_{1}\right\}=\mathbb{J}_{2},\left\{\mathbb{J}_{0}, \mathbb{J}_{2}\right\}=-\mathbb{J}_{1},\left\{\mathbb{J}_{1}, \mathbb{J}_{2}\right\}=-\mathbb{J}_{0}$. All three generators Poisson-commute with the integrals $\mathcal{J}_{i}$ and $\mathcal{S}_{i}^{+}$, i.e.

$$
\left\{\mathcal{Y}_{i}^{ \pm}, \mathbb{J}_{\mu}\right\}=0, \quad \mu=0,1,2 .
$$

The Poisson brackets of $\mathcal{K}$ with $\Theta_{0}$ and $\Theta_{i}$ generate the Grassmann-odd dynamical integrals,

$$
\Omega_{0}=\rho_{0}-2 \Theta_{0} t, \quad \Omega_{i}=\rho_{i}-2 \Theta_{i} t
$$


where

$$
\rho_{0}=\vec{r} \cdot \vec{\xi}, \quad \vec{\rho}=\vec{r} \times \vec{\xi}-\xi_{0} \vec{r} .
$$

The Grassmann-odd quantities $\left(\rho_{0}, \vec{\rho}\right)$ are transformed by the chiral spin integral $\mathcal{S}_{i}^{+}$and total angular momentum $\mathcal{J}_{i}$ in the same way as $\left(\xi_{0}, \vec{\xi}\right)$. The $\Omega_{0}$ and $\Omega_{i}$ are, respectively, the scalar and vector integrals with respect to $\mathcal{J}_{i},\left\{\mathcal{J}_{i}, \Omega_{0}\right\}=0,\left\{\mathcal{J}_{i}, \Omega_{j}\right\}=\epsilon_{i j k} \Omega_{k}$, while with respect to the chiral spin vector $\mathcal{S}_{i}^{+}$they transform in the same way as the integrals $\Theta_{0}$ and $\Theta_{i}$. Hence the dynamical integrals $\Omega_{a}$ have the Poisson bracket relations of the form (3.19) with the $s o(4)$ generators $\mathcal{Y}_{i}^{ \pm}$. The Poisson brackets of $\Theta_{a}$ and $\Omega_{a}$ with the $s o(2,1)$ generators are

$$
\begin{gathered}
\left\{\mathcal{D}, \Theta_{a}\right\}=\Theta_{a}, \quad\left\{\mathcal{D}, \Omega_{a}\right\}=-\Omega_{a}, \\
\left\{\mathcal{H}, \Theta_{a}\right\}=0, \quad\left\{\mathcal{H}, \Omega_{a}\right\}=-2 \Theta_{a}, \\
\left\{\mathcal{K}, \Theta_{a}\right\}=2 \Omega_{a}, \quad\left\{\mathcal{K}, \Omega_{a}\right\}=0 .
\end{gathered}
$$

The Poisson bracket relations between the Grassmann-odd integrals are

$$
\left\{\Theta_{a}, \Theta_{b}\right\}=-i \delta_{a b} \mathcal{H}, \quad\left\{\Omega_{a}, \Omega_{b}\right\}=-i \delta_{a b} \mathcal{K},
$$

and

$$
\begin{gathered}
\left\{\Theta_{0}, \Omega_{0}\right\}=-i \mathcal{D}, \quad\left\{\Theta_{i}, \Omega_{j}\right\}=i \epsilon_{i j k}\left(\mathcal{J}_{k}-4 \mathcal{S}_{k}^{+}\right)-i \delta_{i j} \mathcal{D}, \\
\left\{\Theta_{0}, \Omega_{i}\right\}=-i\left(\mathcal{J}_{i}+2 \mathcal{S}_{i}^{+}\right), \quad\left\{\Theta_{i}, \Omega_{0}\right\}=i\left(\mathcal{J}_{i}+2 \mathcal{S}_{i}^{+}\right) .
\end{gathered}
$$

Thus, the set $\mathcal{H}, \mathcal{J}_{i}, \mathcal{S}_{i}^{+}, \mathcal{D}, \mathcal{K}$ of even, and the set $\Theta_{a}, \Omega_{a}$ of odd integrals together form a closed Lie superalgebra. To identify it, we represent the Poisson bracket relations (3.19), (3.29) and (3.30) in a compact form:

$$
\left\{\mathcal{Y}_{i}^{ \pm}, \Upsilon_{a}\right\}=\frac{1}{2} t_{a b}^{ \pm i} \Upsilon_{b}, \quad\left\{\Theta_{a}, \Omega_{b}\right\}=-i \mathcal{D} \delta_{a b}+2 i\left(\alpha t_{a b}^{-i} \mathcal{Y}_{i}^{-}-(1+\alpha) t_{a b}^{+i} \mathcal{Y}_{i}^{+}\right)
$$

where $\Upsilon_{a}=\Theta_{a}, \Omega_{a}$,

$$
t_{a b}^{ \pm i}=-t_{b a}^{ \pm i}, \quad t_{0 j}^{ \pm i}= \pm \delta_{j}^{i}, \quad t_{j k}^{ \pm i}=\epsilon_{i j k},
$$

and $\alpha=1 / 2$. We conclude that the nine bosonic integrals $\mathcal{H}, \mathcal{K}, \mathcal{D}, \mathcal{Y}_{i}^{+}$and $\mathcal{Y}_{i}^{-}$, and the eight fermionic integrals $\Theta_{a}$ and $\Omega_{a}$ generate the superconformal $D(2,1 ; \alpha)$ symmetry [41, 42, 43, 35. 443 with $\alpha=1 / 2$. The three bosonic integrals $\mathcal{H}, \mathcal{K}, \mathcal{D}$ together with a pair of fermionic integrals $\Theta_{a}, \Omega_{a}$ with fixed $a$ generate one of the four copies of the $\operatorname{ssp}(1 \mid 2)$ Lie superalgebra. As a minimal generating set one can take, for example, the odd integrals $\Theta_{0}$ and $\Omega_{0}$, and the even integrals $\mathcal{S}_{1}^{+}$and $\mathcal{S}_{2}^{+}$. Their successive Poisson brackets fully reproduce the described superconformal Lie superalgebra. The quadratic Casimir element of the Lie superalgebra $D(2,1 ; \alpha)$ can be presented in the form [45, 46]

$$
\mathcal{C}=\tilde{\mathcal{Y}}_{i} \tilde{\mathcal{Y}}_{i}+\alpha \mathcal{Y}_{i}^{-} \mathcal{Y}_{i}^{-}-(1+\alpha) \mathcal{Y}_{i}^{+} \mathcal{Y}_{i}^{+}+\frac{i}{2} \Theta_{a} \Omega_{a}
$$

\footnotetext{
${ }^{3}$ Superalgebra $D(2,1 ; \alpha)$ has an automorphism associated with permutations of the three $s o(3)$ subalgebras, which are generated by $\mathcal{Y}_{i}^{+}, \mathcal{Y}_{i}^{-}$and $\tilde{\mathcal{Y}}_{i}$, where $\tilde{\mathcal{Y}}_{1}=i \mathbb{J}_{1}, \tilde{\mathcal{Y}}_{2}=i \rrbracket_{2}, \tilde{\mathcal{Y}}_{3}=\mathbb{J}_{0}$. At the parameter level the automoprphism corresponds to the dihedral group $D_{3}$ generated by the transformations $\alpha \rightarrow-(1+\alpha)$ and $\alpha \rightarrow \alpha^{-1}$. As a result, the superalgebras $D(2,1 ; \lambda)$ with $\lambda=\alpha^{ \pm 1},-(1+\alpha)^{ \pm 1}$ and $-\left(\frac{\alpha}{1+\alpha}\right)^{ \pm 1}$ are isomorphic [42, and the superalgebra we have here can be identified as the $D(2,1 ; \alpha)$ with the parameter $\alpha$ taking any value from the set $\{-3,-3 / 2,-2 / 3,-1 / 3,1 / 2,2\}$.
} 
In our case $\tilde{\mathcal{Y}}_{1}=i \rrbracket_{1}, \tilde{\mathcal{Y}}_{2}=i \mathbb{J}_{2}, \tilde{\mathcal{Y}}_{3}=\mathbb{J}_{0}, \tilde{\mathcal{Y}}_{i} \tilde{\mathcal{Y}}_{i}=\mathbb{J}_{0}^{2}-\mathbb{J}_{1}^{2}-\mathbb{J}_{2}^{2}=\frac{1}{4}\left(\mathcal{H} \mathcal{K}-\mathcal{D}^{2}\right)$, and one can easily check that at $\alpha=1 / 2$ the $\mathcal{C}$ given by Eq. (3.33) Poisson commutes with all the even and odd generators $\mathcal{H}, \mathcal{K}, \mathcal{D}, \mathcal{Y}_{i}^{ \pm}$and $\Theta_{a}, \Omega_{a}$.

We have here $\frac{i}{2} \Theta_{a} \Omega_{a}=(\vec{L}+\nu \vec{n}) \cdot \overrightarrow{\mathcal{S}}^{-}$, and the quantum analog of the last nilpotent term in $(3.33)$ is

$$
\frac{i}{4}\left[\hat{\Theta}_{a}, \hat{\Omega}_{a}\right]=\hat{\mathcal{L}}_{\sigma}+\frac{3}{2}, \quad \text { where } \quad \hat{\mathcal{L}}_{\sigma} \equiv \mathcal{T}_{-}(1 \otimes(\hat{\vec{L}}+\nu \vec{n}) \cdot \vec{\sigma})
$$

This is a nontrivial integral for the spin- $1 / 2$ subsystem with Hamiltonian $\hat{H}_{-}=\mathcal{T}_{-} \hat{\mathcal{H}}$, see below. It satisfies the relation $\hat{\mathcal{L}}_{\sigma}\left(\hat{\mathcal{L}}_{\sigma}+2\right)=\left(\hat{\overrightarrow{\mathcal{J}}}^{2}-3 / 4\right) \mathcal{T}_{-}$. A rather natural question at this point is whether the system possesses a fermionic type integral which (like the Grassmann-even integral $i \Theta_{a} \Omega_{a} / 2$ ) quantum mechanically would be the square root of the integral $\hat{\vec{J}}^{2}$ (possibly shifted for an additive constant) but without a chiral projector factor. Now we shall show that such a Grassmann-odd integral indeed exists, and that it is associated with the conserved LaplaceRunge-Lenz vector of the superextended system.

To that end consider the Grassmann-odd scalar quantity

$$
\Xi_{0}=-\left(\vec{L}+\frac{2}{3} \overrightarrow{\mathcal{S}}\right) \cdot \vec{\xi}
$$

One can check that it satisfies the Poisson-bracket relation

$$
\left\{\Xi_{0}, \Xi_{0}\right\}=-i\left(\overrightarrow{\mathcal{J}}^{2}-\nu^{2}\right),
$$

and that it is an integral of motion, $\left\{\Xi_{0}, \mathcal{H}\right\}=0$. The Poisson bracket of (3.34) with the chiral spin vector $\mathcal{S}_{i}^{+}$generates then three more integrals of motion, which form a Grassman vector with respect to the total angular momentum,

$$
\vec{\Xi}=\vec{\xi} \times \vec{L}+\xi_{0}(\vec{L}+2 \overrightarrow{\mathcal{S}})
$$

With respect to the chiral spin vector, the integrals $\Xi_{a}$ have properties similar to those of $\Theta_{a}$ and $\Omega_{a}$,

$$
\left\{\mathcal{S}_{i}^{+}, \Xi_{0}\right\}=\frac{1}{2} \Xi_{i}, \quad\left\{\mathcal{S}_{i}^{+}, \Xi_{j}\right\}=\frac{1}{2}\left(\epsilon_{i j k} \Xi_{k}-\delta_{i j} \Xi_{0}\right)
$$

There is, however, an essential difference in comparison with the Grassmann-odd integrals $\Theta_{a}$ and $\Omega_{a}$. One can calculate the Poisson brackets of $\Xi_{0}$ with $\Xi_{i}$ by using the first relation in (3.37) and employing the graded Jacobi identities. Since the Poisson bracket of $\mathcal{S}_{i}^{+}$with the right hand side in (3.35) is nonzero, the integrals $\Xi_{0}$ and $\Xi_{i}$ possess nontrivial Poisson bracket relations,

$$
\left\{\Xi_{0}, \Xi_{i}\right\}=2 i\left(\overrightarrow{\mathcal{S}}^{+} \times \overrightarrow{\mathcal{J}}\right)_{i}
$$

The presence of the quadratic in integrals $\overrightarrow{\mathcal{J}}$ and $\overrightarrow{\mathcal{S}}^{+}$expressions on the right hand sides of (3.38) and (3.35) means that the extension of the set of generators of the superconformal Lie algebra $D(2,1 ; \alpha=1 / 2)$ by the odd integral $\Xi_{0}$ transforms it into a nonlinear superalgebra, in which the parameter $\nu^{2}$ plays a role of the central charge. This is not surprising since such a nonlinearity characterizes the symmetry algebras of systems with a conserved Laplace-Runge-Lenz vector. The nonlinearity originates from the particular form of the integral (3.34) : it is cubic in the phase space variables $4 \Pi_{i}, r_{j}$ and $\xi_{a}$.

\footnotetext{
${ }^{4} \mathrm{~A}$ nonlinear superalgebraic structure associated with the squared total angular momentum appears also in the supersymmetrized charge-monopole system, see [10, 19, 20, 27.
} 
The Poisson brackets between $\Xi_{i}$ and $\Xi_{j}$ can also be computed by using relations (3.37) and employing the graded Jacobi identities. Again, we get a nonlinear (quadratic in the integrals $\mathcal{J}_{i}$ and $\mathcal{S}_{j}^{+}$) Poisson bracket relation,

$$
\left\{\Xi_{i}, \Xi_{j}\right\}=i \delta_{i j}\left(\nu^{2}-\left(\overrightarrow{\mathcal{J}}-2 \overrightarrow{\mathcal{S}}^{+}\right)^{2}\right)+4 i \mathcal{S}_{i}^{+} \mathcal{S}_{j}^{+}-2 i\left(\mathcal{S}_{i}^{+} \mathcal{J}_{j}+\mathcal{S}_{j}^{+} \mathcal{J}_{i}\right)
$$

The scalar integral $\Xi_{0}$ Poisson-commutes with two other scalar Grassmann-odd integrals,

$$
\left\{\Theta_{0}, \Xi_{0}\right\}=\left\{\Omega_{0}, \Xi_{0}\right\}=0 .
$$

On the other hand, we have nontrivial Poisson bracket relations

$$
\begin{gathered}
\left\{\Theta_{i}, \Xi_{0}\right\}=-\left\{\Theta_{0}, \Xi_{i}\right\}=i \mathcal{G}_{i}, \quad\left\{\Omega_{i}, \Xi_{0}\right\}=-\left\{\Omega_{0}, \Xi_{i}\right\}=i \mathcal{R}_{i}, \\
\left\{\Xi_{i}, \Theta_{j}\right\}=-i \epsilon_{i j k} \mathcal{G}_{k}, \quad\left\{\Xi_{i}, \Omega_{j}\right\}=-i \epsilon_{i j k} \mathcal{R}_{k} .
\end{gathered}
$$

This provides us with a generalization of the Laplace-Runge-Lenz vector integral (2.25) and its associated dynamical integral (2.36),

$$
\begin{gathered}
\overrightarrow{\mathcal{G}}=\vec{\Pi} \times\left(\overrightarrow{\mathcal{J}}-\overrightarrow{\mathcal{S}}^{+}\right)+\vec{\Pi} \times \overrightarrow{\mathcal{S}}^{-}+\frac{2 \nu}{r}\left(\overrightarrow{\mathcal{S}}^{-}-\frac{\vec{r}}{r}\left(\vec{r} \cdot \overrightarrow{\mathcal{S}}^{-}\right)\right), \\
\overrightarrow{\mathcal{R}}=\vec{r} \times\left(\overrightarrow{\mathcal{J}}-\overrightarrow{\mathcal{S}}^{+}+\overrightarrow{\mathcal{S}}^{-}\right)-2 \overrightarrow{\mathcal{G} t} .
\end{gathered}
$$

The supersymmetrized Laplace-Runge-Lenz vector (3.43) can be written in the form

$$
\overrightarrow{\mathcal{G}}=\vec{\Pi} \times \overrightarrow{\mathcal{Y}}^{-}-\frac{i}{2} \vec{\xi} \times \vec{\Theta}+i \frac{\nu}{2 r^{3}} \vec{\rho} \times \vec{\rho} .
$$

It Poisson-commutes with the chiral spin vector and supercharges $\Theta_{a}$,

$$
\left\{\mathcal{G}_{i}, \mathcal{S}_{j}^{+}\right\}=\left\{\mathcal{G}_{i}, \Theta_{a}\right\}=0,
$$

while the brackets with the conformal symmetry generators are

$$
\left\{\mathcal{H}, \mathcal{G}_{i}\right\}=0, \quad\left\{\mathcal{D}, \mathcal{G}_{i},\right\}=\mathcal{G}_{i}, \quad\left\{\mathcal{K}, \mathcal{G}_{i},\right\}=2 \mathcal{R}_{i}
$$

For the dynamical vector integral $\mathcal{R}_{i}$ we have

$$
\left\{\mathcal{R}_{i}, \mathcal{S}_{j}^{+}\right\}=\left\{\mathcal{R}_{i}, \Omega_{a}\right\}=0
$$

and in addition

$$
\left\{\mathcal{H}, \mathcal{R}_{i}\right\}=-2 \mathcal{G}_{i}, \quad\left\{\mathcal{D}, \mathcal{R}_{i},\right\}=-\mathcal{R}_{i}, \quad\left\{\mathcal{K}, \mathcal{R}_{i},\right\}=0
$$

We also have Lie type Poisson bracket relations

$$
\begin{gathered}
\left\{\Omega_{0}, \mathcal{G}_{i}\right\}=\Xi_{i}, \quad\left\{\Omega_{i}, \mathcal{G}_{j},\right\}=\epsilon_{i j k} \Xi_{k}-\delta_{i j} \Xi_{0}, \\
\left\{\Theta_{0}, \mathcal{R}_{i}\right\}=-\Xi_{i}, \quad\left\{\Theta_{i}, \mathcal{R}_{j},\right\}=-\epsilon_{i j k} \Xi_{k}+\delta_{i j} \Xi_{0} .
\end{gathered}
$$

It is worth noting here, that although in (3.49) the $\Xi_{a}$ are generated via Poisson brackets of the dynamical odd integrals $\Omega_{a}$ and the true integrals $\mathcal{G}_{i}$, they are true Grassmann integrals. This happens since the time-dependent term $-2 t \Theta_{a}$ in $\Omega_{a}$ Poisson-commutes with $\mathcal{G}_{i}$. 
The Poisson brackets of $\mathcal{G}_{i}$ and $\mathcal{R}_{i}$ with $\Xi_{a}$ are quadratic polynomials in the integrals,

$$
\begin{gathered}
\left\{\Xi_{0}, \mathcal{G}_{i}\right\}=(\overrightarrow{\mathcal{J}} \times \vec{\Theta})_{i}, \quad\left\{\Xi_{0}, \mathcal{R}_{i}\right\}=(\overrightarrow{\mathcal{J}} \times \vec{\Omega})_{i}, \\
\left\{\mathcal{G}_{i}, \Xi_{j}\right\}=\delta_{i j}\left(\overrightarrow{\mathcal{J}}-2 \overrightarrow{\mathcal{S}}^{+}\right) \cdot \vec{\Theta}-\Theta_{0} \epsilon_{i j k} \mathcal{J}_{k}+2 \mathcal{S}_{i}^{+} \Theta_{j}-\Theta_{i} \mathcal{J}_{j}, \\
\left\{\mathcal{R}_{i}, \Xi_{j}\right\}=\delta_{i j}\left(\overrightarrow{\mathcal{J}}-2 \overrightarrow{\mathcal{S}}^{+}\right) \cdot \vec{\Omega}-\Omega_{0} \epsilon_{i j k} \mathcal{J}_{k}+2 \mathcal{S}_{i}^{+} \Omega_{j}-\Omega_{i} \mathcal{J}_{j} .
\end{gathered}
$$

The Poisson brackets between the integrals $\mathcal{G}_{i}$ and $\mathcal{R}_{i}$ are also quadratic,

$$
\begin{gathered}
\left\{\mathcal{G}_{i}, \mathcal{G}_{j}\right\}=-\mathcal{H} \epsilon_{i j k} \mathcal{J}_{k}-\frac{i}{2} \epsilon_{i j k}(\vec{\Theta} \times \vec{\Theta})_{k} \\
\left\{\mathcal{R}_{i}, \mathcal{R}_{j}\right\}=-\mathcal{K} \epsilon_{i j k} \mathcal{J}_{k}-\frac{i}{2} \epsilon_{i j k}(\vec{\Omega} \times \vec{\Omega})_{k} \\
\left\{\mathcal{G}_{i}, \mathcal{R}_{j}\right\}=\delta_{i j}\left(\nu^{2}-2 \overrightarrow{\mathcal{J}}^{2}+4 \overrightarrow{\mathcal{J}} \cdot \overrightarrow{\mathcal{S}}^{+}+i \vec{\Omega} \cdot \vec{\Theta}\right)+\frac{i}{2}\left(\Theta_{i} \Omega_{j}+\Theta_{j} \Omega_{i}\right)+\mathcal{J}_{i} \mathcal{J}_{j}-2\left(\mathcal{S}_{i}^{+} \mathcal{J}_{j}+\mathcal{S}_{j}^{+} \mathcal{J}_{i}\right) \\
-\epsilon_{i j k}\left(\mathcal{D} \mathcal{J}_{k}+\frac{i}{2}(\vec{\Theta} \times \vec{\Omega})_{k}\right)
\end{gathered}
$$

With these relations we obtain a closed, nonlinear (quadratic) superconformal algebra extended by the Laplace-Runge-Lenz vector $\overrightarrow{\mathcal{G}}$, the associated Grassmann-even dynamical vector integral $\overrightarrow{\mathcal{R}}$, and by the Grassmann-odd integrals $\Xi_{a}$.

Let us now discuss shortly some aspects of the quantum version of the described supersymmetric structure, in which the Poisson brackets between the Grassmann-odd generators become anticommutators of the corresponding quantum fermionic operators, while the brackets between the Grassmann-even with Grassmann-even or Grassmann-odd generators become commutators between their quantum counterparts.

The quantum analog of the Hamiltonian $(3.16)$ is the matrix $4 \times 4$ operator having a blockdiagonal form,

$$
\hat{\mathcal{H}}=\hat{\vec{\Pi}}^{2}+\frac{\nu^{2}}{r^{2}}-\frac{2 \nu}{r^{3}} \mathcal{T}_{-}(1 \otimes \vec{\sigma} \cdot \vec{r})=\left(\begin{array}{cc}
\hat{H}_{+} & 0 \\
0 & \hat{H}_{-}
\end{array}\right)
$$

where the $2 \times 2$ Hamiltonians are $\hat{H}_{+}=\hat{\vec{\Pi}}^{2}+\nu^{2} / r^{2}$ and $\hat{H}_{-}=\hat{\vec{\Pi}}^{2}+\nu^{2} / r^{2}-2 \nu(\vec{\sigma} \cdot \vec{r}) / r^{3}$. Note that the chiral operator $\hat{H}_{+}$is proportional to the $2 \times 2$ identity matrix. This can be attributed to the self-duality of the dyon field. The conserved total angular momentum operator $\hat{\vec{J}}=\hat{\vec{J}}+\hat{\overrightarrow{\mathcal{S}}}$, contains the spinless part $\hat{\vec{J}}=\vec{r} \times \hat{\vec{\Pi}}-\nu \vec{n}$ and the spin operator $\hat{\overrightarrow{\mathcal{S}}}=1 \otimes \frac{1}{2} \vec{\sigma}$. By standard arguments, the parameter $\nu$ undergoes the Dirac quantization: at the quantum level it can take only integer or half-integer values, i.e. $\nu=n / 2, n \in \mathbb{Z}[9$, 47].

The operator $\hat{H}_{+}$represents two identical copies of the Hamiltonian operator of the spinless system discussed in the previous section, and the integral nature of the chiral spin operator $\hat{\mathcal{S}}^{+}=\mathcal{T}_{+}\left(1 \otimes \frac{1}{2} \vec{\sigma}\right)$ is then obvious. The diagonal operator $\hat{\mathcal{S}}_{3}^{+}$distinguishes the upper and lower components of this doubled spinless system, while the operators $\hat{\mathcal{S}}_{1}^{+} \pm i \hat{\mathcal{S}}_{2}^{+}$transform them one into another in an obvious way. The operator $\hat{H}_{-}$can be interpreted as the Pauli type Hamiltonian of the charged spin-1/2 particle of gyromagnetic ratio 4 in the combined field of the magnetic monopole and scalar potential $\nu^{2} / r^{2}$. 
The scalar and vector quantum supercharges for the extended system (3.57) have a blockantidiagonal form

$$
\hat{\Theta}_{a}=\frac{1}{\sqrt{2}}\left(\begin{array}{cc}
0 & \hat{Q}_{a} \\
\hat{Q}_{a}^{\dagger} & 0
\end{array}\right)
$$

where

$$
\hat{Q}_{0}=-i \vec{\sigma} \cdot \hat{\vec{\Pi}}+\frac{\nu}{r}, \quad \hat{\vec{Q}}=-i \vec{\sigma} \hat{Q}_{0}=-i\left(\hat{\vec{\Pi}} \times \vec{\sigma}+\frac{\nu}{r} \vec{\sigma}\right)-\hat{\vec{\Pi}} .
$$

They commute with the Hamiltonian (3.57) and anticommute with the diagonal grading operator $\Gamma$ in (3.3), which itself is an additional quantum integral of motion of the bosonic nature. Note that $\hat{Q}_{a}$ and $\hat{Q}_{a}^{\dagger}$ are the Darboux intertwining operators :

$$
\hat{Q}_{a} \hat{H}_{-}=\hat{H}_{+} \hat{Q}_{a}, \quad \hat{Q}_{a}^{\dagger} \hat{H}_{+}=\hat{H}_{-} \hat{Q}_{a}^{\dagger} .
$$

These relations are equivalent to the condition of commutativity of supercharges $\hat{\Theta}_{a}$ and $i \Gamma \hat{\Theta}_{a}$ with $\hat{\mathcal{H}}$. As in the case of the one-dimensional supersymmetric quantum mechanics, they allow us to relate the eigenstates $\psi_{+}$and $\psi_{-}$of the Hamiltonians $\hat{H}_{+}$and $\hat{H}_{-}$of the quantum subsystems, $\psi_{+} \propto \hat{Q}_{a} \psi_{-}, \psi_{-} \propto \hat{Q}_{a}^{\dagger} \psi_{+}$.

The superconformal generators $\hat{\Omega}_{a}$ have a block-antidiagonal form similar to that in (3.58), with $\hat{Q}_{0}$ replaced by $-i \vec{\sigma} \cdot \vec{r}-2 \hat{Q}_{0} t$, and $\hat{\vec{Q}}$ replaced by $-i \vec{r} \times \vec{\sigma}-\vec{r}-2 \hat{\vec{Q}} t$. The quantum analogs $\hat{\Xi}_{a}$ of the supercharges (3.34) and (3.36) have the same block-antidiagonal form, but with the scalar operator $\hat{Q}_{0}$ replaced by $i(\vec{\sigma} \cdot \hat{\vec{L}}+1)$, and the vector operator $\hat{\vec{Q}}$ replaced by $-(\hat{\vec{L}}+\vec{\sigma})-i \hat{\vec{L}} \times \vec{\sigma}$, where $\hat{\vec{L}}=\vec{r} \times \hat{\vec{\Pi}}$. Note that in the anticommutator $\left[\hat{\Xi}_{0}, \hat{\Xi}_{0}\right]_{+}=\hat{\vec{J}}^{2}-\nu^{2}+1 / 4$, there appears a quantum correction term $\hbar^{2} / 4$.

The Laplace-Runge-Lenz vector operator of the supersymmetric quantum system is

$$
\hat{\overrightarrow{\mathcal{G}}}=\hat{\vec{G}}+\mathcal{T}_{-} \cdot 1 \otimes\left(\hat{\vec{\Pi}} \times \vec{\sigma}+\frac{\nu}{r}(\vec{\sigma}-\vec{n}(\vec{\sigma} \cdot \vec{n}))\right),
$$

where $\hat{\vec{G}}$ is a Hermitian spinless Laplace-Runge-Lenz vector,

$$
\hat{\vec{G}}=\frac{1}{2}(\hat{\vec{\Pi}} \times \hat{\vec{J}}-\hat{\vec{J}} \times \hat{\vec{\Pi}})=-\hat{\vec{J}} \times \hat{\vec{\Pi}}+i \hat{\vec{\Pi}}
$$

It commutes with the supercharges $\hat{\Theta}_{a}$, and thus with the Hamiltonian $\hat{\mathcal{H}}$. The quantum analog of the related, dynamical vector integral (3.44) is

$$
\hat{\overrightarrow{\mathcal{R}}}=\frac{1}{2}(\vec{r} \times \hat{\vec{J}}-\hat{\vec{J}} \times \vec{r})-\mathcal{T}_{-}(1 \otimes \vec{\sigma} \times \vec{r})-2 \hat{\vec{G}} t .
$$

In correspondence with the classical properties, it satisfies the commutation relation $[\hat{\overrightarrow{\mathcal{R}}}, \hat{\mathcal{H}}]=2 i \hat{\overrightarrow{\mathcal{G}}}$.

\section{Summary, concluding remarks and outlook}

Let us summarize the obtained nonlinear superalgebraic structure of the spinning charged particle in the background of the self-dual dyon.

A very particular role in the supersymmetric structure is played by the chiral spin vector $\overrightarrow{\mathcal{S}}^{+}$, whose origin is rooted in the self-dual nature of the background electromagnetic field. This bosonic, Grassmann-even vector integral commutes with all other basic bosonic integrals, except 
the total angular momentum vector $\overrightarrow{\mathcal{J}}$. On the other hand, all the fermionic, Grassmann-odd integrals are grouped into the three irreducible representations with respect to the Poisson bracket action, or, commutator in the quantum case, of $\overrightarrow{\mathcal{S}}^{+}$on them. The rotational symmetry associated with $\overrightarrow{\mathcal{J}}$ is due to a spherical symmetry of the magnetic and dual to it electric fields of the dyon. The sets of the integrals $\mathcal{Y}_{i}^{-}=\mathcal{J}_{i}-\mathcal{S}_{i}^{+}$and $\mathcal{Y}_{i}^{+}=\mathcal{S}_{i}^{+}$generate the $s o(3) \oplus s o(3)=s o(4)$ Lie subalgebra.

One can distinguish the three entangled supersymmetry substructures in the system, each of which can be related to its corresponding basic bosonic integral.

i) The Hamiltonian $\mathcal{H}$ is a supersymmetric generalization of the spinless Hamiltonian (2.18), which possesses conformal symmetry and reveals a hidden partially free dynamics. The peculiar dynamics of the spinless system is encoded in the presence of the conserved Laplace-RungeLenz vector $\vec{G}$ and the associated dynamical integral $\vec{R}$, which can be related to the deformed Galilei symmetry. Quantum mechanically, the square root of $\hat{\mathcal{H}}$ is the scalar supercharge $\hat{\Theta}_{0}$, whose classical Grassmann-odd analog $\Theta_{0}$ Poisson-commutes with itself for $-i \mathcal{H}$. The Poisson bracket of $\Theta_{0}$ with the chiral spin vector integral $\overrightarrow{\mathcal{S}}^{+}$generates three more integrals, $\Theta_{i}$, which form a vector $\vec{\Theta}$ with respect to the total angular momentum integral $\overrightarrow{\mathcal{J}}$. Each $\Theta_{i}$, like $\Theta_{0}$, is a square root of $\mathcal{H}$ : together these four supercharges $\Theta_{a}$ generate the $N=4$ supersymmetry: $\left\{\Theta_{a}, \Theta_{b}\right\}=-i \delta_{a b} \mathcal{H},\left\{\Theta_{a}, \mathcal{H}\right\}=0$. The Poisson bracket of $\Theta_{i}$ with $\mathcal{S}_{j}^{+}$is a linear combination of $\Theta_{0}$ and $\Theta_{k}$. On the quantum level the interplay between self-duality and extended supersymmetry has been emphasized in a more general context (without further assumptions) in [48, see also [40] and [26].

ii) The dynamical integral $\mathcal{K}$, which explicitly depends on time, generates the special conformal transformations. Its bracket with the Hamiltonian $\mathcal{H}$ produces the generator of dilatations $\mathcal{D}$ as a further dynamical integral. The integrals $\mathcal{H}, \mathcal{K}, \mathcal{D}$ Poisson-commute with the integrals $\mathcal{J}_{i}$ and $\mathcal{S}_{i}^{+}$, and generate a $s o(2,1)$ symmetry. The classical analog of the square root of the quantum operator $\hat{\mathcal{K}}$ corresponds to the Grassmann-odd dynamical scalar integral $\Omega_{0}$. The Poisson brackets of $\Omega_{0}$ with the integrals $\mathcal{S}_{i}^{+}$generate three more dynamical integrals $\Omega_{i}$, which form a vector with respect to $\overrightarrow{\mathcal{J}}$. The set $\Omega_{a}, a=0, i$, has the same transformational properties with respect to $\mathcal{S}_{i}^{+}$as the Grassmann odd integrals $\Theta_{a}$. The Grassmann-odd dynamical integrals $\Omega_{a}$ Poisson commute with $\mathcal{K}$, and together they generate the sub-superalgebra, similar to that generated by $\Theta_{a}$ and $\mathcal{H}:\left\{\Omega_{a}, \Omega_{b}\right\}=-i \delta_{a b} \mathcal{K},\left\{\Omega_{a}, \mathcal{K}\right\}=0$.

The dynamical integrals $\Omega_{a}$ are eigenstates of $\mathcal{D}$ with eigenvalue -1 , which means $\left\{\mathcal{D}, \Omega_{a}\right\}=$ $-\Omega_{a}$. Similarly $\mathcal{K}$ is an eigenstate with eigenvalue -2 and $\mathcal{H}$ is an eigenstate with eigenvalue +2 . Accordingly one finds that the conserved supercharges $\Theta_{a}$ are eigenstates of $\mathcal{D}$ of eigenvalue +1 .

The superalgebraic structures outlined in i) and ii) are entangled via via Poisson brackets. The bracket of $\mathcal{H}$ with the dynamical integrals $\Omega_{a}$ produces the integrals $\Theta_{a}$, while the bracket of $\mathcal{K}$ with the integrals $\Theta_{a}$ generates $\Omega_{a}$. The Poisson brackets between the supercharges $\Theta_{a}$ and superconformal charges $\Omega_{a}$ produce linear combinations of $\mathcal{D}, \mathcal{J}_{i}$ and $\mathcal{S}_{i}^{+}$, from which these even integrals can be completely reconstructed.

The usual integrals $\mathcal{H}, \mathcal{Y}_{i}^{ \pm}, \Theta_{a}$ (which do not depend explicitly on time) together with the dynamical integrals $\mathcal{K}, \mathcal{D}$, and $\Omega_{a}$ generate the exceptional simple Lie superconformal algebra $D(2,1 ; \alpha)$ with $\alpha=1 / 2$. This Lie superalgebra of order 17 has the quadratic Casimir $\mathcal{C}=$ $\frac{1}{4}\left(\mathcal{H} \mathcal{K}-\mathcal{D}^{2}\right)+\frac{1}{2} \overrightarrow{\mathcal{Y}}^{-2}-\frac{3}{2} \overrightarrow{\mathcal{Y}}^{+2}+\frac{i}{2} \Theta_{a} \Omega_{a}$. To the last Grassmann-even nilpotent term corresponds the quantum operator $\frac{i}{4}\left[\hat{\Theta}_{a}, \hat{\Omega}_{a}\right]=\hat{\mathcal{L}}_{\sigma}+3 / 2$ with $\hat{\mathcal{L}}_{\sigma} \equiv \mathcal{T}_{-}[1 \otimes(\hat{\vec{L}}+\nu \vec{n}) \cdot \vec{\sigma}]$ which is a nontrivial integral for the spin- $1 / 2$ subsystem $\hat{H}_{-}$. The quantum bosonic integral $\hat{\mathcal{L}}_{\sigma}$ satisfies the quadratic 
relation $\hat{\mathcal{L}}_{\sigma}\left(\hat{\mathcal{L}}_{\sigma}+2\right)=\mathcal{T}_{-}\left(\hat{\overrightarrow{\mathcal{J}}}^{2}-3 / 4\right)$.

iii) Similarly as for $\hat{\mathcal{H}}$ and $\hat{\mathcal{K}}$, there exists a anti-diagonal fermionic square root for the conserved operator $\hat{\overrightarrow{\mathcal{J}}}^{2}$. Its classical analog is the Grassmann-odd scalar integral $\Xi_{0}$, which satisfies the Poisson bracket relation $\left\{\Xi_{0}, \Xi_{0}\right\}=-i\left(\overrightarrow{\mathcal{J}}^{2}-\nu^{2}\right)$. The bracket of $\Xi_{0}$ with the chiral spin vector $\overrightarrow{\mathcal{S}}^{+}$produces the Grassmann odd vector integral $\vec{\Xi}$, and the set $\Xi_{a}$ is transformed by $\mathcal{S}_{i}^{+}$in the same way as the integrals $\Theta_{a}$ and $\Omega_{a}$. However, $\Xi_{i}$ has nonzero Poisson brackets with $\Xi_{0}$, and the brackets of $\Xi_{a}$ with $\Xi_{b}$ turn out to be quadratic in the total angular momentum $\mathcal{J}_{i}$ and the chiral spin vector $\mathcal{S}_{i}^{+}$. On the other hand, in contrast with the Grassmann-odd integrals $\Theta_{a}$ and $\Omega_{a}$, the integrals $\Xi_{a}$, like $\mathcal{J}_{i}$ and $\mathcal{S}_{i}^{+}$, Poisson-commute with the $s o(2,1)$ generators $\mathcal{K}$ and $\mathcal{D}$, which means that they are $s o(2,1)$ scalars.

The Poisson brackets of $\Xi_{a}$ with $\Theta_{b}$ generate the Laplace-Runge-Lenz vector integral $\mathcal{G}_{i}$. Analogously, the brackets of $\Xi_{a}$ with $\Omega_{b}$ produce the dynamical vector integral $\mathcal{R}_{i}$ associated with $\mathcal{G}_{i}, \frac{\partial}{\partial t} \mathcal{R}_{i}=\mathcal{G}_{i}$. In correspondence with this, $\mathcal{G}_{i}$ and $\mathcal{R}_{i}$ are eigenvectors of $\mathcal{D}$ with eigenvalues +1 and -1 , respectively. The Poisson bracket of $\mathcal{G}_{i}$ with $\mathcal{K}$ leads to $\mathcal{R}_{i}$, while $\mathcal{R}_{i}$ Poissoncommutes with $\mathcal{K}$. The Hamiltonian $\mathcal{H}$ acts on these vector integrals in the opposite way: it Poisson-commutes with $\mathcal{G}_{i}$, and its bracket with the dynamical integral $\mathcal{R}_{i}$ yields the integral $\mathcal{G}_{i}$. These bosonic vector integrals Poisson-commute with the chiral spin vector $\mathcal{S}_{i}^{+}$. At the same time, the vector $\mathcal{G}_{i}$ Poisson-commutes with $\Theta_{a}$, while its brackets with $\Omega_{a}$ produce integrals $\Xi_{a}$. Analogously, the dynamical integral $\mathcal{R}_{i}$ Poisson commutes with $\Omega_{a}$, and generates the Grassmannodd integrals $\Xi_{a}$ via the brackets with $\Theta_{a}$. The Poisson brackets of $\mathcal{G}_{i}$ and $\mathcal{R}_{i}$ with $\Xi_{a}$, and between $\mathcal{G}_{i}$ and $\mathcal{R}_{i}$ yields quadratic expressions of the other integrals. So, the extension of the set of the generators of the superconformal symmetry $D(2,1 ; \alpha=1 / 2)$ by the Grassmann-odd and Grassmann-even integrals $\Xi_{a}$ and $\mathcal{G}_{i}, \mathcal{R}_{i}$ transforms the Lie superalgebra into a nonlinear, quadratic superalgebra.

It is worth noting that starting with any one of the set of the ten integrals $\Xi_{a}, \mathcal{G}_{i}$ and $\mathcal{R}_{i}$, one can generate the complete set of integrals by taking Poisson brackets with the generators of $D(2,1 ; \alpha=1 / 2)$, for instance,

$$
\Xi_{0} \stackrel{\mathcal{S}_{i}^{+}}{\longmapsto} \Xi_{i} \stackrel{\Theta_{j}}{\longmapsto} \mathcal{G}_{i} \stackrel{\mathcal{K}}{\longmapsto} \mathcal{R}_{i} \stackrel{\Theta_{j}}{\longmapsto} \Xi_{0}, \Xi_{i}
$$

Then, as the minimal set of the integrals generating all the quadratic superalgebra via the Poisson brackets one can take one of the Grassmann-odd integrals $\Theta_{a}$, one of the Grassmann-odd dynamical integrals $\Omega_{b}$, two components of the chiral spin vector $\overrightarrow{\mathcal{S}}^{+}$, and finally one of the odd or even integrals $\Xi_{a}$ or $\mathcal{J}_{i}, \mathcal{R}_{i}$.

Quantum mechanically the system has the additional integral $\Gamma=\gamma_{5}=-\gamma_{0} \gamma_{1} \gamma_{2} \gamma_{3}=\tau_{3} \otimes 1$. It commutes with quantum analogs of all the Grassmann-even integrals, but anticommutes with the quantum analogs of all the Grassmann-odd integrals. It is identified as the $\mathbb{Z}_{2}$-grading operator of the quantum supersymmetric structure. The multiplication of any of the fermionic Hermitian generators $\hat{\Phi}_{\mu}$ from the set $\left(\hat{\Theta}_{a}, \hat{\Omega}_{a}, \hat{\Xi}_{a}\right)$ by $i \gamma_{5}$ gives a new Hermitian fermionic integral, and in an algebraic way doubles the set of them. As a result, instead of the nonlinear, quadratic superalgebra consisting of 12 fermionic integrals, we end up with a superalgebra with 24 fermionic generators satisfying the commutation relations $\left[\Gamma, \hat{\Phi}_{s \mu}\right]=-2 i \epsilon_{s s^{\prime}} \hat{\Phi}_{s^{\prime} \mu}$, where $s, s^{\prime}=1,2, \hat{\Phi}_{1 \mu}=$ $\hat{\Phi}_{\mu}$ and $\hat{\Phi}_{2 \mu}=i \Gamma \hat{\Phi}_{1 \mu}$. The linear combinations $\hat{\Phi}_{ \pm \mu}=\frac{1}{2}\left(\hat{\Phi}_{1 \mu} \mp i \hat{\Phi}_{2 \mu}\right)=\mathcal{T}_{ \pm} \hat{\Phi}_{\mu}$ with $\left(\hat{\Phi}_{+\mu}\right)^{\dagger}=\hat{\Phi}_{-\mu}$, define chiral fermionic generators, see Eq. (3.58), and the integrals $\hat{\Xi}_{ \pm a}$ are Darboux generators that intertwine the spin-independent (doubled) Schrödinger operator $\hat{H}_{+}$and spin-dependent Pauli operator $\hat{H}_{-}$. This property has a natural explanation if one views $\hat{H}_{ \pm}$as parts of a 
squared Euclidean Dirac operator for selfdual background fields [48]. Analogously, the $\hat{\Omega}_{ \pm a}$ are Darboux intertwiners for the operators $\hat{\mathcal{K}}_{+}$and $\hat{\mathcal{K}}_{-}, \hat{\mathcal{K}}_{ \pm}=\mathcal{T}_{ \pm} \hat{\mathcal{K}}$, which are generators of the special conformal symmetries of the corresponding spinless and spin-1/2 quantum subsystems.

On the other hand, at the classical level the quantity $-2 \xi_{0} \xi_{1} \xi_{2} \xi_{3}=\frac{1}{3} \overrightarrow{\mathcal{S}}^{+2}$ corresponds to the operator $\gamma_{5}$. This, however, is an Grassmann-even integral, whose quantum analog includes a multiplicative factor $\hbar^{2}$ in contrast with the quantum analog of $\xi_{a}$ to be proportional to $\sqrt{\hbar}$. A multiplication of this nilpotent Grassmann-even integral by any of the classical Grassmann-odd integral produces zero, and we have no classical analog for the quantum fermionic integrals $\hat{\Phi}_{2 \mu}$. Alternatively, as a classical analog of $\gamma_{5}$, one could try to introduce an independent Grassmann scalar variable $\xi_{5}$ with the only nontrivial Poisson bracket $\left\{\xi_{5}, \xi_{5}\right\}=-i$. In this case multiplication of classical integrals $\Theta_{a}, \Omega_{a}$ and $\Xi_{a}$ by $i \xi_{5}$ will give new classically nontrivial integrals. However, they will be of the Grassmann-even, bosonic nature. The described problems associated with the introduction of the classical analog for the matrix $\gamma_{5}$ are well known and were discussed in a different context in the literature [49].

Based on relations (2.43) and (2.44) for the spinless bosonic case, one could assume that by a suitable nonlinear (non-local at the quantum level) redefinition of the even, $\mathcal{G}_{i}$ and $\mathcal{R}_{i}$, and the odd, $\Xi_{a}$, generators of the nonlinear superconformal symmetry it can be reduced to some linear superextension of the $D(2,1 ; \alpha=1 / 2)$. Let us show that such a linearization, however, is impossible.

Nonlinear (quadratic) algebraic relations (3.54) and (3.55) are linearized by a redefinition $\mathcal{G}_{i} \rightarrow \breve{\mathcal{G}}_{i}, \mathcal{R}_{i} \rightarrow \breve{\mathcal{R}}_{i}$

$$
\breve{\mathcal{G}}_{i}=\frac{1}{\sqrt{\mathcal{H}}} \mathcal{G}_{i}+\frac{1}{2 \mathcal{H}}(\vec{\Theta} \times \vec{\Theta})_{i}, \quad \breve{\mathcal{R}}_{i}=\frac{1}{\sqrt{\mathcal{K}}} \mathcal{R}_{i}+\frac{1}{2 \mathcal{K}}(\vec{\Omega} \times \vec{\Omega})_{i}
$$

These vectors satisfy, particularly, the Lie-Poisson algebraic relations

$$
\left\{\breve{\mathcal{G}}_{i}, \breve{\mathcal{G}}_{j}\right\}=-\epsilon_{i j k} \mathcal{J}_{k}, \quad\left\{\breve{\mathcal{G}}_{i}, \Theta_{j}\right\}=i \epsilon_{i j k} \Theta_{k}, \quad\left\{\breve{\mathcal{G}}_{i}, \mathcal{H}\right\}=\left\{\breve{\mathcal{G}}_{i}, \mathcal{D}\right\}=\left\{\breve{\mathcal{G}}_{i}, \Theta_{0}\right\}=0,
$$

and

$$
\left\{\breve{\mathcal{R}}_{i}, \breve{\mathcal{R}}_{j}\right\}=-\epsilon_{i j k} \mathcal{J}_{k}, \quad\left\{\breve{\mathcal{R}}_{i}, \Omega_{j}\right\}=i \epsilon_{i j k} \Omega_{k}, \quad\left\{\breve{\mathcal{R}}_{i}, \mathcal{K}\right\}=\left\{\breve{\mathcal{R}}_{i}, \mathcal{D}\right\}=\left\{\breve{\mathcal{R}}_{i}, \Omega_{0}\right\}=0 .
$$

One can also redefine $\Xi_{a} \rightarrow \breve{\Xi}_{a}$

$$
\breve{\Xi}_{0}=\frac{\Xi_{0}}{\sqrt{\overrightarrow{\mathcal{J}}^{2}-\nu^{2}}}, \quad \breve{\Xi}_{i}=\frac{\Xi_{i}}{\sqrt{\overrightarrow{\mathcal{J}}^{2}-\nu^{2}}}-\frac{2 \breve{\Xi}_{0}}{\overrightarrow{\mathcal{J}}^{2}-\nu^{2}}\left(\overrightarrow{\mathcal{J}} \times \overrightarrow{\mathcal{S}}^{+}\right)_{i} .
$$

These integrals satisfy with the $\mathcal{S}_{i}^{+}$the same relations as the initial odd integrals $\Xi_{a},\left\{\mathcal{S}_{i}^{+}, \breve{\Xi}_{0}\right\}=$ $\frac{1}{2} \breve{\Xi}_{i},\left\{\mathcal{S}_{i}^{+}, \breve{\Xi}_{j}\right\}=\frac{1}{2}\left(\epsilon_{i j k} \breve{\Xi}_{k}-\delta_{i j} \breve{\Xi}_{0}\right)$, but instead of the nonlinear Poisson bracket relations (3.35), (3.38) and (3.39) we get

$$
\left\{\breve{\Xi}_{a}, \breve{\Xi}_{b}\right\}=-i \delta_{a b}
$$

These last Poisson brackets can be treated as a superalgebraic relation with central charge 1 . The brackets

$$
\left\{\Theta_{i}, \breve{\Xi}_{0}\right\}=-\left\{\Theta_{0}, \breve{\Xi}_{i}\right\}=i \frac{\mathcal{G}_{i}}{\sqrt{\overrightarrow{\mathcal{J}}^{2}-\nu^{2}}}-\frac{(\overrightarrow{\mathcal{J}} \times \vec{\Theta})_{i}}{\overrightarrow{\mathcal{J}}^{2}-\breve{\Xi}^{2}}
$$

together with Eq. (4.1) show nevertheless that the redefined integrals generate a more complicated, non-polynomial superalgebra (which is of a non-local nature at the quantum level). 
Analogous non-polynomial structures appear in the Poisson brackets of $\Theta_{a}$ and $\mathcal{H}$ with $\breve{\mathcal{R}}_{i}$, and in those of $\Omega_{a}$ and $\mathcal{K}$ with $\breve{\mathcal{G}}_{i}$. The brackets of $\breve{\mathcal{G}}_{i}$ with $\breve{\mathcal{R}}_{j}$ are also of a non-polynomial form in other integrals. This shows the impossibility of the linearization of the obtained quadratic extension of the superconformal symmetry $D(2,1 ; \alpha=1 / 2)$.

The model we considered possesses only the continuous spectrum with $E>0$. It is known that in the quantum Kepler problem the hidden symmetry associated with the dynamical LaplaceRunge-Lenz vector integral not only determines the energy levels completely, but also the phase shifts [50]. One could naturally expect that the hidden supersymmetry we discussed here may also be helpful for analyzing the scattering characteristics of the quantum system. Investigation of this problem is outside of the scope of the present work and deserves a separate consideration.

The dynamics of the boson (spinless) sector of the system we studied corresponds to the special bosonic dynamics of the $D(2,1 ; \alpha)$ model with $\alpha^{2}=1 / 4$ investigated by Ivanov, Krivonos and Lechtenfeld in [25]. The approach of [25] is completely different, however, that allowed the authors to obtain the general $D(2,1 ; \alpha)$ models. Namely, in [25] the $N=4$ superconformal mechanics realizing the $D(2,1 ; \alpha)$ symmetry with arbitrary values of the parameter $\alpha$ was constructed in the $N=4, d=1$ superspace. It was shown then that the bosonic sector of the system describes a conformally invariant nonlinear sigma model, which at the two particular values $\alpha=+1 / 2$ and $\alpha=-1 / 2$ reduces to the system (2.18) 5 . We have shown that the superextension of the system, which corresponds to an (anti)self-dual dyon background, is described by a qudratically extended Lie superalgebra $D(2,1 ; \alpha)$ with the parameter $\alpha=1 / 2$. This belongs to the set of the values $\{-3,-3 / 2,-2 / 3,-1 / 3,1 / 2,2\}$, which can be related by the superalgebra automorphism $D_{3}$, see footnote 3 . The case of $\alpha=-1 / 2$, also appearing in special bosonic dynamics in [25], is not included in the indicated set. As it is clear, particularly, from the form of the $D(2,1 ; \alpha)$ Casimir (3.33), the case $\alpha=-1 / 2$ (related by the $D_{3}$ automorphism to the cases $\alpha=-2$ and $\alpha=1)$ is essentially different. For this value of the parameter $\alpha$ the Casimirs of the two so(3) subalgebras enter with the same "weight" into the $D(2,1 ; \alpha)$ Casimir. Therefore, it seems to be very interesting to investigate another special case $\alpha=-1 / 2$ of the $D(2,1 ; \alpha)$ model [25] from the viewpoint of nonlinear extension of superconformal symmetry associated with a presence in the bosonic sector of the Laplace-Runge-Lenz vector and its dynamical integral counterpart.

As it was already noted in the introduction, for one-dimensional quantum systems with soliton potentials the hidden free nature of the systems reveals itself in two related ways. Namely, such systems are characterized by zero reflection coefficient, and they possess the Lax-Novikov higher order differential operator as a nontrivial integral. The latter is a Darboux-dressed form of the free particle momentum operator, and its differential order is fixed by the number of solitons 'hidden' in the potential. The hidden free nature also shows up in a supersymmetric extension of a reflectionless system. Instead of the two supercharges which we have in ordinary, superextended systems with two superpartner Hamiltonians, the solitonic superextended systems admit four supercharges. Together with the two bosonic integrals associated with the Lax-Novikov integrals, they generate a nontrivial nonlinear superalgeba [8]. The 3D quantum system we studied here also reveals a hidden, partially free dynamics. The system (2.18) is characterized by a vanishing scattering angle for all values of $J, J^{2} \geq \nu^{2}$, and this property is reminiscent of the vanishing reflection coefficient in $1 \mathrm{D}$ solitonic systems. Thus it may be interesting to investigate whether the system we studied here possesses yet an additional hidden nonlinear supersymmetric structure

\footnotetext{
${ }^{5}$ Special bosonic dynamics is interpreted in 25] by means of Eq. (5.2) there as that describing electrically charged particle in spherically symmetric electric and magnetic fields of the (non-(anti)self-dual) form $\mathcal{E}_{i}=\nu^{2} r_{i} / r^{4}$ and $\mathcal{B}_{i}=-\nu r_{i} / r^{3}$, cf. [40, 17] and our discussion in Section 3 .
} 
associated with a partially free nature of its dynamics. This is suggested by the property of the $1 \mathrm{D}$ conformal quantum mechanical model with $a / x^{2}$ potential. It has a hidden, bosonized supersymmetric structure for special values of the strength $a$ [51].

Acknowledgements. We thank Sergey Fedoruk and Olaf Lechtenfeld for helpful comments. The work has been partially supported by FONDECYT Grant 1130017, and by DICYT (USACH), and by DFG-Grants Wi 777/11 and GRK 1523. MSP and AW are grateful, respectively, to the Universities of Jena and Santiago de Chile for hospitality.

\section{References}

[1] W. Pauli, "Über das Wasserstoffspektrum vom Standpunkt der neuen Quantenmechanik," Z. Physik 36, 336 (1926).

[2] V. Fock, "Zur Theorie des Wasserstoffatoms", Z. Phys. 98, 145 (1935).

[3] V. Bargmann, "Zur Theorie des Wasserstoffatoms: Bemerkungen zur gleichnamigen Arbeit von V. Fock," Z. Phys. 99, 576 (1936).

[4] H. Goldstein, "Prehistory of the "Runge-Lenz" vector," Am. J. Phys. 43, 737 (1975); "More on the prehistory of the Laplace or RungeLenz vector," Am. J. Phys. 44, 1123 (1975).

[5] A. Kirchberg, J. D. Lange, P. A. G. Pisani and A. Wipf, "Algebraic solution of the supersymmetric hydrogen atom in d-dimensions," Annals Phys. 303, 359 (2003) [hep-th/0208228.

[6] A. Wipf, A. Kirchberg and J. D. Lange, "Algebraic solution of the supersymmetric hydrogen atom," Bulg. J. Phys. 33, 206 (2006) [hep-th/0511231].

[7] S. P. Novikov, S.V. Manakov, L. P. Pitaevskii, and V. E. Zakharov, Theory of Solitons (Plenum, New York, 1984).

[8] A. Arancibia, J. M. Guilarte and M. S. Plyushchay, "Effect of scalings and translations on the supersymmetric quantum mechanical structure of soliton systems," Phys. Rev. D 87, 045009 (2013) arXiv:1210.3666 [math-ph]]; "Fermion in a multi-kink-antikink soliton background, and exotic supersymmetry," Phys. Rev. D 88, 085034 (2013) arXiv:1309.1816 [hep-th]].

[9] P. Goddard and D. I. Olive, "Magnetic monopoles in gauge field theories," Rept. Prog. Phys. 41, 1357 (1978).

[10] M. S. Plyushchay, "Monopole Chern-Simons term: Charge monopole system as a particle with spin," Nucl. Phys. B 589, 413 (2000) hep-th/0004032; "Free conical dynamics: Charge-monopole as a particle with spin, anyon and nonlinear fermion-monopole supersymmetry," Nucl. Phys. Proc. Suppl. 102, 248 (2001) hep-th/0103040].

[11] D. Zwanziger, "Exactly soluble nonrelativistic model of particles with both electric and magnetic charges," Phys. Rev. 176, 1480 (1968).

[12] H. V. Mcintosh and A. Cisneros, "Degeneracy in the presence of a magnetic monopole," J. Math. Phys. 11, 896 (1970).

[13] E. Witten, "Dyons of charge e $\theta / 2 \pi$," Phys. Lett. B 86, 283 (1979). 
[14] R. Jackiw, "Dynamical symmetry of the magnetic monopole," Annals Phys. 129, 183 (1980).

[15] E. D'Hoker and L. Vinet, "Constants of motion for a spin 1/2 particle in the field of a dyon," Phys. Rev. Lett. 55, 1043 (1985).

[16] E. D'Hoker and L. Vinet, "Hidden symmetries and accidental degeneracy for a spin 1/2 particle in the field of a dyon," Lett. Math. Phys. 12, 71 (1986).

[17] E. D'Hoker and L. Vinet, “Dynamical supersymmetry of the magnetic monopole and the $1 / r^{2}$ potential," Commun. Math. Phys. 97 (1985) 391.

[18] L. G. Feher and P. A. Horvathy, "Non-relativistic scattering of a spin-1/2 particle off a selfdual monopole," Mod. Phys. Lett. A 3, 1451 (1988) arXiv:0903.0249 [hep-th]]; L. Feher, P. A. Horvathy and L. O'Raifeartaigh, "Separating the dyon system," Phys. Rev. D 40,666 (1989); "Applications of chiral supersymmetry for spin fields in selfdual backgrounds," Int. J. Mod. Phys. A 4, 5277 (1989) [arXiv:0903.2920 [hep-th]]; F. Bloore and P. A. Horvathy, "Helicity supersymmetry of dyons," J. Math. Phys. 33, 1869 (1992) hep-th/0512144].

[19] G. W. Gibbons, R. H. Rietdijk and J. W. van Holten, "SUSY in the sky," Nucl. Phys. B 404, 42 (1993) hep-th/9303112; F. De Jonghe, A. J. Macfarlane, K. Peeters and J. W. van Holten, "New supersymmetry of the monopole," Phys. Lett. B 359, 114 (1995) hep-th/9507046.

[20] M. S. Plyushchay, "On the nature of fermion-monopole supersymmetry," Phys. Lett. B 485, 187 (2000) hep-th/0005122.

[21] P. A. Horvathy, A. J. Macfarlane and J. W. van Holten, "Monopole supersymmetries and the Biedenharn operator," Phys. Lett. B 486, 346 (2000) hep-th/0006118.

[22] G. Papadopoulos, "Conformal and superconformal mechanics," Class. Quant. Grav. 17, 3715 (2000) hep-th/0002007.

[23] J. A. de Azcarraga, J. M. Izquierdo and A. J. Macfarlane, "Hidden supersymmetries in supersymmetric quantum mechanics," Nucl. Phys. B 604, 75 (2001) [hep-th/0101053].

[24] I. I. Cotaescu and M. Visinescu, "Runge-Lenz operator for Dirac field in Taub - NUT background," Phys. Lett. B 502, 229 (2001) hep-th/0101163.

[25] E. Ivanov, S. Krivonos and O. Lechtenfeld, "New variant of $N=4$ superconformal mechanics,” JHEP 0303, 014 (2003) hep-th/0212303.

[26] E. Ivanov and O. Lechtenfeld, "N=4 supersymmetric mechanics in harmonic superspace," JHEP 0309, 073 (2003) hep-th/0307111.

[27] C. Leiva and M. S. Plyushchay, "Nonlinear superconformal symmetry of a fermion in the field of a Dirac monopole," Phys. Lett. B 582, 135 (2004) hep-th/0311150.

[28] S. Bellucci, A. Nersessian and A. Yeranyan, "Hamiltonian reduction and supersymmetric mechanics with Dirac monopole," Phys. Rev. D 74, 065022 (2006) hep-th/0606152.

[29] S. G. Avery and J. Michelson, "Mechanics and quantum supermechanics of a monopole probe including a Coulomb potential," Phys. Rev. D 77, 085001 (2008) arXiv:0712.0341 [hep-th]]. 
[30] J. P. Ngome, P. A. Horvathy and J. W. van Holten, "Dynamical supersymmetry of spin particle-magnetic field interaction," J. Phys. A 43, 285401 (2010) [arXiv:1003.0137 [hep-th]].

[31] D. Anninos, T. Anous, F. Denef, G. Konstantinidis and E. Shaghoulian, "Supergoop dynamics," JHEP 1303, 081 (2013) arXiv:1205.1060 [hep-th]].

[32] S. Krivonos, A. Nersessian and V. Ohanyan, "Multi-center MICZ-Kepler system, supersymmetry and integrability," Phys. Rev. D 75, 085002 (2007) hep-th/0611268.

[33] S. -T. Hong, J. Lee, T. H. Lee and P. Oh, "A complete solution of a constrained system: SUSY monopole quantum mechanics," JHEP 0602, 036 (2006) hep-th/0511275.

[34] V. de Alfaro, S. Fubini and G. Furlan, "Conformal invariance in quantum mechanics," Nuovo Cim. A 34, 569 (1976).

[35] J. Michelson and A. Strominger, "The geometry of (super)conformal quantum mechanics," Commun. Math. Phys. 213, 1 (2000) [hep-th/9907191].

[36] P. Claus, M. Derix, R. Kallosh, J. Kumar, P. K. Townsend and A. Van Proeyen, "Black holes and superconformal mechanics," Phys. Rev. Lett. 81, 4553 (1998) hep-th/9804177.

[37] C. Leiva and M. S. Plyushchay, "Conformal symmetry of relativistic and nonrelativistic systems and AdS/CFT correspondence," Annals Phys. 307, 372 (2003) hep-th/0301244.

[38] S. Fedoruk, E. Ivanov and O. Lechtenfeld, “OSp(4|2) superconformal mechanics,” JHEP 0908, 081 (2009) arXiv:0905.4951 [hep-th]].

[39] S. Fedoruk, E. Ivanov and O. Lechtenfeld, "New $D(2,1 ; \alpha)$ mechanics with spin variables," JHEP 1004, 129 (2010) arXiv:0912.3508 [hep-th]].

[40] M. de Crombrugghe and V. Rittenberg, "Supersymmetric quantum mechanics," Annals Phys. 151, 99 (1983).

[41] J. Van Der Jeugt, "Irreducible representations of the exceptional Lie superalgebras $D(2,1 ; \alpha), "$ J. Math. Phys. 26, 913 (1985).

[42] L. Frappat, P. Sorba and A. Sciarrino, "Dictionary on Lie superalgebras," hep-th/9607161.

[43] R. Britto-Pacumio, J. Michelson, A. Strominger and A. Volovich, "Lectures on superconformal quantum mechanics and multiblack hole moduli spaces," hep-th/9911066.

[44] E. Ivanov, S. Krivonos and O. Lechtenfeld, " $N=4, d=1$ supermultiplets from nonlinear realizations of D(2,1; $\alpha)$," Class. Quant. Grav. 21, 1031 (2004) hep-th/0310299.

[45] T. Matsumoto and S. Moriyama, "An exceptional algebraic origin of the AdS/CFT Yangian symmetry," JHEP 0804, 022 (2008) arXiv:0803.1212 [hep-th]].

[46] S. Fedoruk, E. Ivanov and O. Lechtenfeld, "Superconformal mechanics," J. Phys. A 45, 173001 (2012) arXiv:1112.1947 [hep-th]].

[47] P. A. M. Dirac, "Quantized singularities in the electromagnetic field," Proc. Roy. Soc. Lond. A 133, 60 (1931). 
[48] A. Kirchberg, J. D. Lange and A. Wipf, "Extended supersymmetries and the Dirac operator," Annals Phys. 315, 467 (2005) hep-th/0401134.

[49] M. S. Plyushchay, "Pseudoclassical description of the massive spinning particle in $d$ dimensions," Mod. Phys. Lett. A 8, 937 (1993); J. Gamboa and M. Plyushchay, "Classical anomalies for spinning particles," Nucl. Phys. B 512, 485 (1998) hep-th/9711170.

[50] D. Zwanziger, "Algebraic calculation of nonrelativistic Coulomb phase shifts,", J. Math. Phys. 8, 1858 (1967).

[51] F. Correa, M. A. del Olmo and M. S. Plyushchay, "On Hidden broken nonlinear superconformal symmetry of conformal mechanics and nature of double nonlinear superconformal symmetry," Phys. Lett. B 628, 157 (2005) hep-th/0508223. 